\title{
La superposición de cartografía histórica como método de análisis morfológico y toma de decisiones urbanísticas. Ibagué, Colombia, 1935-2016
}

\author{
Sobreposição de cartografia histórica como método de análise morfológica e \\ tomada de decisões de planejamento, Ibagué, Colômbia, 1935-2016 \\ Overlapping historical cartography as a method of morphological analysis and \\ taking planning decisions. Ibague, Colombia, 1935-2016
}

Andrés Francel

Universidad del Tolima, Ibagué, Colômbia

\section{Resumen}

Ibagué es una ciudad intermedia colombiana en cuyo centro existe una zona socialmente deprimida y comercialmente efervescente. Para solucionar sus problemáticas se realizó una propuesta de rediseño urbanístico dentro del marco de los planes parciales nacionales, finalmente incorporados a los principios de planeación del Ministerio de Vivienda en el año 2012. Sin embargo, el plan no se ha ejecutado, por lo cual tiene una condición de idealidad y probabilidad, común a los planeamientos urbanísticos desde 1935. Para comprender las convergencias y divergencias entre estos planes y la realidad urbanística, se realizó un análisis del sector utilizando el método histórico-artístico en su componente objetual ajustado para el análisis de morfologías. La superposición cartográfica para la identificación de tendencias evidencia el continuo desconocimiento de las dinámicas históricas y el desacierto en los procesos de planeación. La investigación aporta criterios de análisis que pueden ayudar a la incorporación de estudios morfológicos históricos para la toma de decisiones urbanísticas en sectores similares y en ciudades que presentan superposiciones entre las políticas nacionales y el desarrollo municipal.

Palabras clave: Heteromorfismo urbano. Cicatriz urbana. Costura urbana.

\section{Resumo}

No centro de Ibagué, uma cidade média da Colômbia, há uma área comercialmente agitada e socialmente decadente. Para resolver os problemas da cidade, uma proposta de redesenho urbana foi feita no âmbito de planos parciais nacionais, eventualmente incorporados aos princípios de planejamento do Ministério da Habitação, em 2012. No entanto, o plano não foi implementado, por isso há uma condição da idealidade e probabilidade comum à planificação urbana desde 1935. Para entender as semelhanças e as diferenças entre esses planos e a realidade urbana, uma análise do setor foi realizada usando o método histórico-artístico em seu componente objetal ajustado para analisar morfologias. O mapa de sobreposição para identificar tendências evidencia a ignorância continuada da dinâmica histórica e o erro de cálculo no processo de planejamento. A pesquisa fornece critérios de análise que podem ajudar a incorporar estudos histórico-morfológicos para a tomada de decisões de planejamento em setores similares e em cidades que têm sobreposições entre as políticas nacionais e o desenvolvimento municipal.

Palavras-chave: Heteromorfismo urbano. Cicatriz urbana. Costura urbana.

AF es arquiteto, doctor en Historia, e-mail: aefranceld@ut.edu.co 


\section{Abstract}

Ibagué is a Colombian medium-sized city in which the center is a commercially vibrant and socially depressed area. To solve their problems a proposal of urban redesign within the framework of national partial plans was made eventually incorporated into the planning principles the Ministry of Housing in 2012. However, the plan has not been implemented, as it has a condition of ideality and probability, common to urban planning since 1935. To understand the similarities and differences between these plans and the urban reality, a sector analysis was performed using the historic-artistic method in its object-based component adjusted for analyzing morphologies. The map overlay for identifying trends evidence the continued ignorance of the historical dynamics and miscalculation in planning processes. This research provides analysis criteria that can help incorporating historical morphological studies in order to make planning decisions in similar sectors and in cities that have overlaps between national policies and municipal development as a cause of conflicts.

Keywords: Urban heteromorphism. Urban scar. Urban couture.

\section{Introducción}

Los planes parciales son estrategias contemporáneas de intervención urbanística en Colombia que permiten redefinir pequeñas zonas como instrumentos de apoyo a los Planes de Ordenamiento Territorial (POT) (Angulo Posse, 2011), cuya representación en Ibagué se encuentra en el Plan Parcial de la Calle 19, que ha sido aprobado pero no ejecutado (Ibagué, 2013). El área determinada para la intervención urbanística incluye importantes objetos arquitectónicos y urbanísticos como la plaza de mercado de la 21, el terminal de transportes (antigua estación del ferrocarril), el parque público más grande del centro la ciudad y una quebrada canalizada bajo tierra. El desarrollo de esta zona comenzó alrededor de 1904 y se consolidó hacia 1940 (Francel, 2013). Sin embargo, la planimetría más antigua para su análisis data de 1931 y las propuestas para su intervención se encuentran en el plan urbanístico Ibagué Futuro de 1935 (Francel, 2014). En sucesivos años se han generado planteamientos para mejorar las condiciones del sector pero ninguna se ha implementado en su totalidad, motivo por el cual se propuso la identificación histórica de las dinámicas territoriales cuyo análisis derivara en interpretaciones sobre el desarrollo histórico de diversas zonas urbanas y las posibilidades de actuación en el planeamiento futuro de la ciudad.

\section{Materiales y métodos}

Se aplicó el método histórico-artístico en su vertiente objetual, consistente en "la definición de estilos a través de la observación y de la comparación" (Moreno
Martín, 2014), al cual se realizó un ajuste conceptual para analizar morfologías urbanas en lugar de estilos arquitectónicos y sus incidencias socio-espaciales (Delgado, 2006). Como sustento se utilizó el método filológico-documental para recomponer con la precisión posible el "[...] contexto en el que fue creada, recibida, utilizada/modificada y, en ocasiones, destruida" la traza urbana (Moreno Martín, 2014, p. 6). Para ello, se revisaron los archivos históricos de la ciudad, con lo cual se obtuvo la totalidad de la cartografía generada sobre la ciudad. La herramienta de acompañamiento para el proceso fue la restitución planimétrica mediante la vectorización (calco digital), el retoque digital y las gráficas analíticas (modelación de datos) (Van der Maas, 2011). Para suplir los vacíos documentales se aplicó el levantamiento oral de fuentes vivas, lo cual permitió la aproximación a criterios de validación de la información mediante la lectura crítica de las fuentes y el contraste de la información.

El primer paso para abordar el tema investigado fue la búsqueda de fuentes primarias que permitieran determinar con exactitud las condiciones de la zona estudiada desde 1935. Para ello, se realizó una selección preliminar de actas notariales en el Archivo Histórico Municipal, cuyo contenido definió las medidas de la parcelación de la zona, los materiales de las construcciones, el porcentaje de ocupación y el estado de las vías.

Simultáneamente, se realizó la búsqueda e identificación de la cartografía en el Archivo Histórico Municipal. La mayoría de los planos encontrados son inéditos y no estaban catalogados. Por ello se procedió con su conservación digital, consistente en su captura fotográfica, vectorización o calco digital mediante 
AutoCAD, ArchiCAD e Illustrator y retoque digital mediante Photoshop. El producto de esta recuperación fue entregado en medio magnético al Archivo Histórico Municipal para facilitar su consulta pública.

El tercer momento consistió en la interpretación del informe del plano urbano de 1935. Para determinar la exactitud de sus descripciones, se compararon con la información consignada en las actas notariales y en la cartografía histórica. Al final de este proceso correlacional, fueron claras las características de la zona en el momento inicial del recorte cronológico. Como complemento, se generaron nuevos planos para llenar los vacíos gráficos del informe original y de este modo establecer una comparación directa con la morfología y la arquitectura actual.

Se realizó la búsqueda de información fotográfica de la zona en la colección de la biblioteca Darío Echandía del Banco de la República. De las 824 imágenes que la conforman, existe una captura de la calle 19 en 1935 en la cual se observan las condiciones de borde de ciudad, en completa coherencia con las descripciones de las fuentes notariales y cartográficas. Existen otras fotografías en diversos momentos sobre las cuales se realizaron observaciones comparativas que serán descritas en los apartados posteriores.

El establecimiento de los momentos intermedios de planeación se realizó a partir de la exploración de la planoteca de la biblioteca Darío Echandía del Banco de la República. En ella se encuentran las copias de los planos realizados por el Instituto Geográfico Agustín Codazzi (IGAC) cuyos datos clasificatorios permiten identificar si se trataron de momentos de actualización nacional o de un estudio perteneciente al diseño de un plan urbanístico. A estos planos se realizaron las operaciones de recuperación, vectorización y retoque y fueron donados a la biblioteca y al archivo histórico. Como complemento, se realizó un ejercicio de superposición cartográfica, revelado y máscara que será descrito posteriormente. Finalmente, se realizó una revisión bibliográfica en medios análogos y digitales, un levantamiento oral y la generación de una síntesis gráfica que se presentará al final.

\section{Resultados y discusión}

La ciudad en 1935

En 1935, Ibagué tenía 36.000 habitantes (SIDEICO, 1935). El cálculo de su población fue aproximado porque se basó en los censos realizados en 1913
(Notaría Primera, 1913) y 1918 (Notaría Primera, 1918a), junto con el conteo de las cuadras de la ciudad en 1918 (Notaría Primera, 1918b) y el informe de casas de comercio y oficinas particulares (Notaría Primera, 1918c). Sin embargo, presenta una síntesis sobre las condiciones de consolidación de la ciudad hasta la Calle 21, luego de la cual se encontraba la zona de expansión (Tabla 1, Figura 1).

Las condiciones urbanísticas del sector de la quebrada El Sillón se observan en un plano identificado con el número 23, realizado por la firma SIDEICO en 1935 (Figura 2). En él puede observarse la división predial irregular que corresponde con una dinámica de comercio inmobiliario dependiente del libre mercado inmobiliario, no de un plan de desarrollo territorial planeado por el gobierno municipal. Esta característica concuerda con el incremento de la dependencia gubernamental de la tributación privada como mecanismo para compensar la insuficiencia de los fondos estatales (Francel, 2013). Una de sus manifestaciones fue el loteo o parcelación de los bordes de ciudad, para lo cual no existió un énfasis en las condiciones equitativas ni en la coherencia de expansión de la ciudad sino una supeditación a la conveniencia económica.

La ausencia de lineamientos de ordenamiento territorial permitió que el comercio inmobiliario funcionara como un elemento autónomo y aislado, afirmación que se soporta en la comparación con el barrio Belén, de origen particular fundado en 1921, en el cual todos los lotes tenían dimensiones similares y no dependieron de la especulación sino que obedecieron a los principios urbanísticos determinados por la firma urbanizadora Manrique Martin \& Co. (Figura 3) (Francel, 2015). En síntesis, la espontaneidad de la zona de expansión de 1935 fue consecuencia de la concentración del gobierno municipal en los intereses económicos sin criterios urbanísticos.

Tabla 1 - Población estimada para el suministro de agua

\begin{tabular}{lc}
\hline \multicolumn{1}{c}{ Sector } & Habitantes \\
\hline Tanques hasta la Calle 21 & 20.000 \\
Calle 21 y quebrada El Sillón al norte & 8.000 \\
Calle 21 y quebrada El Sillón al sur & 8.000 \\
TOTAL & 36.000 \\
\hline
\end{tabular}

Tabulación: Andrés Francel (2015) Fuente: Informe de SIDEICO (1935:0015). Archivo Histórico Municipal. 


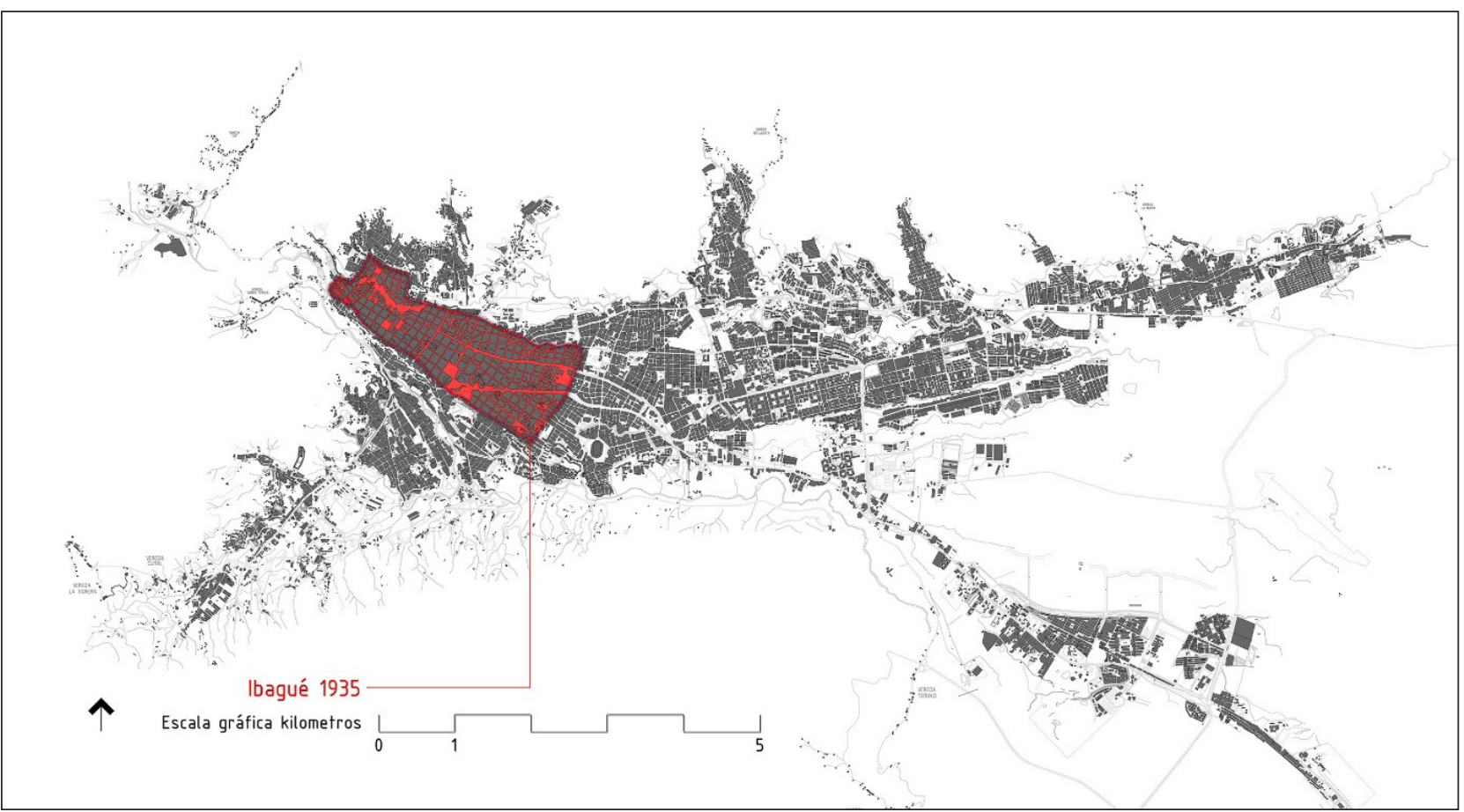

Figura 1 - Extensión de lbagué en 1935 con respecto al plano actual (2015) Fuente: Dibujo por José Alejandro Ojeda (2015).

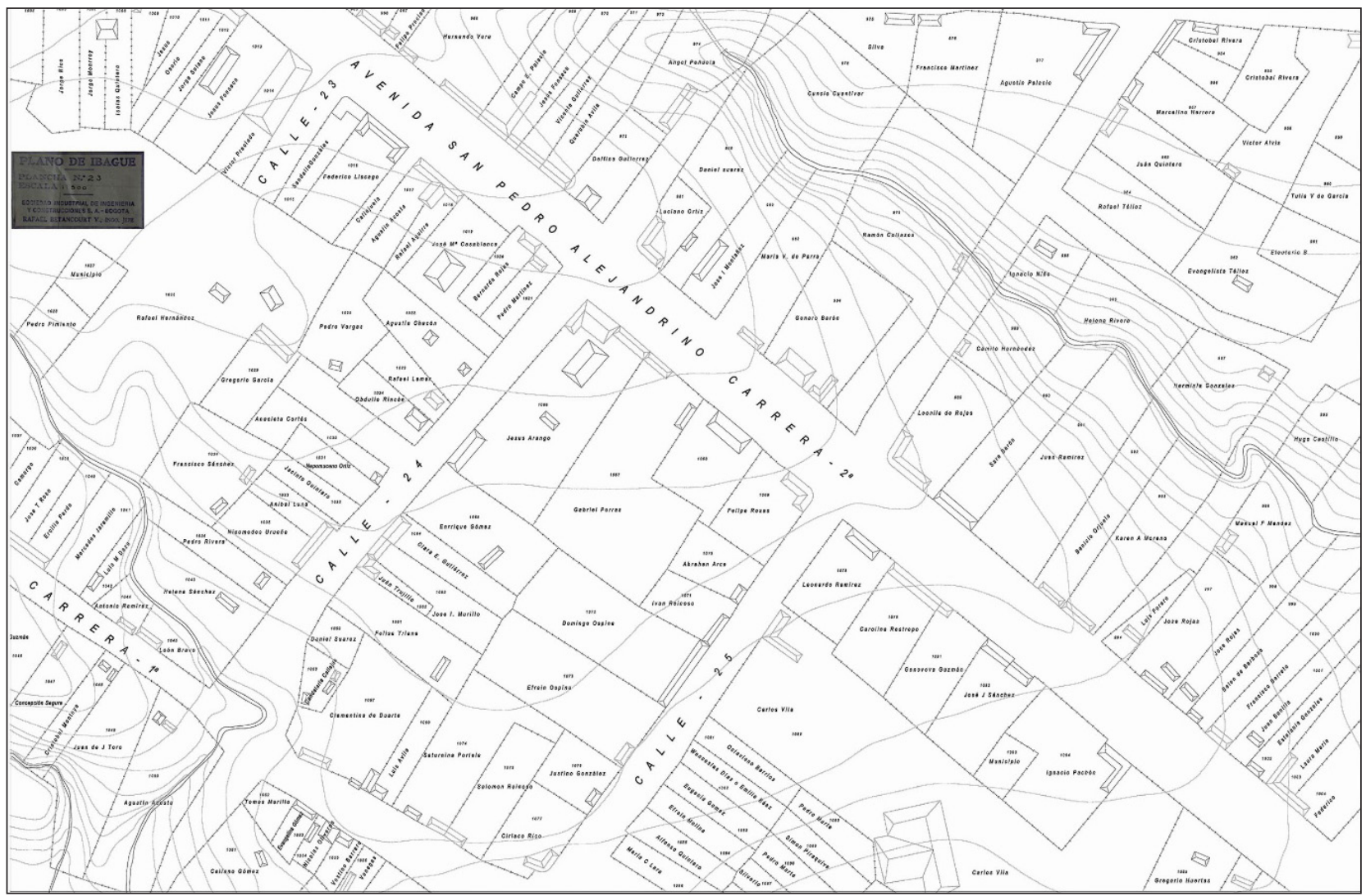

Figura 2 - Plano 23 del informe de SIDEICO (1935)

Fuente: Digitalizado por Zuled Claviijo (2015). 


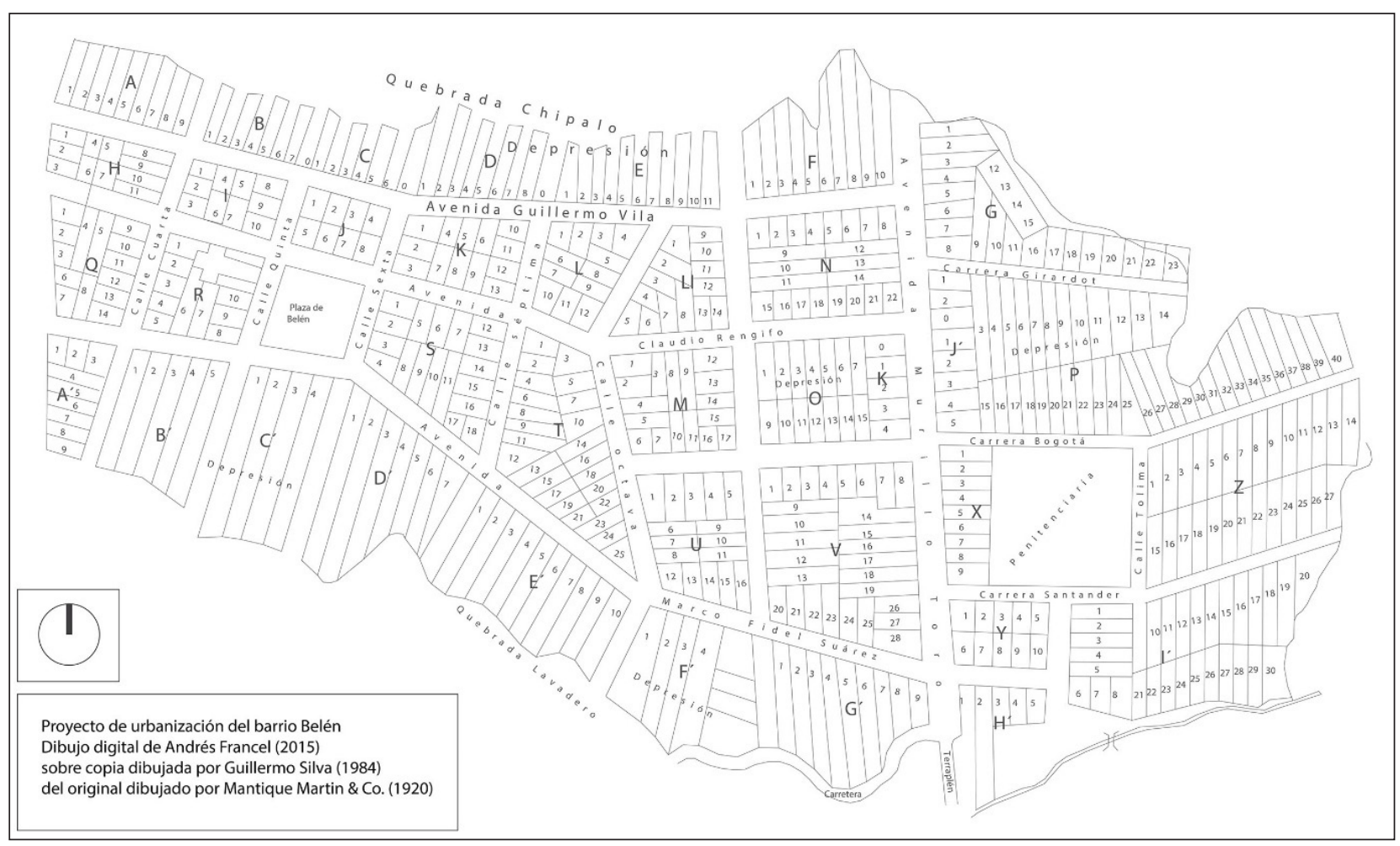

Figura 3 - Plano de urbanización del barrio Belén

Fuente: Elaboración propia (2015) sobre copia dibujada por Guillermo Silva (1984) del original dibujado por Manrique Martin \& Co. (1920).

La segunda característica observada (Figura 2) es la ocupación de los predios en tres formas básicas: a lo largo de la avenida principal como consecuencia del desarrollo comercial, hacia el interior de los predios como evidencia de la explotación agrícola para un adecuado control desde el centro de la parcela y mediante construcciones independientes. Como efecto, no se observa un desarrollo urbano articulado sino una continuidad acorde con la espontaneidad de la división predial.

La tercera característica evidente en el plano 23 es la superposición de la cuadrícula o traza colonial sobre las condiciones geográficas, de modo que esta zona de expansión continuó el damero colonial sin operar variaciones para interpretar los cambios topográficos en las fuentes hídricas. Como consecuencia, el lecho de los afluentes quedó ubicado al interior de las manzanas (Figura 2). La identificación de estas características fue la base para analizar el desarrollo urbano a partir de las variables de discontinuidad (o interrupción de vías) y heteromorfismo (o cambio en la forma del trazado urbano).
Discontinuidad y heteremorfismo urbano

Se revisó toda la cartografía de la ciudad hasta observar el momento de la transformación. Los planos fueron separados en dos categorías. En la primera está el plano de 1931 (Figura 4), el de 1942 (Figura 5), el diagrama de niveles de vida de 1958 (Figura 6) y el Plan Piloto de 1966 (Figura 7). Ellos conforman el cuerpo de estudios iniciales sobre la ciudad, por lo cual se analizan en apartados temáticos individuales. La segunda categoría corresponde a nueve planos entre 1970 y 2014 con características descriptivas similares, cuyo estudio se realizó a partir de superposiciones (Figura 8).

El plano de 1931 (Figura 4) presenta la proyección de las calles de acuerdo con la traza colonial y evidencia transformaciones en sus patrones morfológicos desde una retícula colonial homomórfica (con la misma forma), hacia un modelo más espontáneo heteromórfico (con forma diferente), derivado de la parcelación que realizó el municipio con el propósito de obtener recursos para reinvertirlos en infraestructura. Estas medidas estuvieron vinculadas 
con el proyecto de extensión de las vías férreas en 1920 y la legalización de la urbanización de los terrenos alrededor de la futura estación del ferrocarril (1928) (Francel, 2013, p. 125).
Se identificaron cinco zonas morfológicas en el plano de 1942 a la cuales se asignó numeración en orden izquierda-derecha/arriba-abajo y se identificaron las calles con la letra $\mathrm{C}$ y las carreras con la letra $\mathrm{K}$

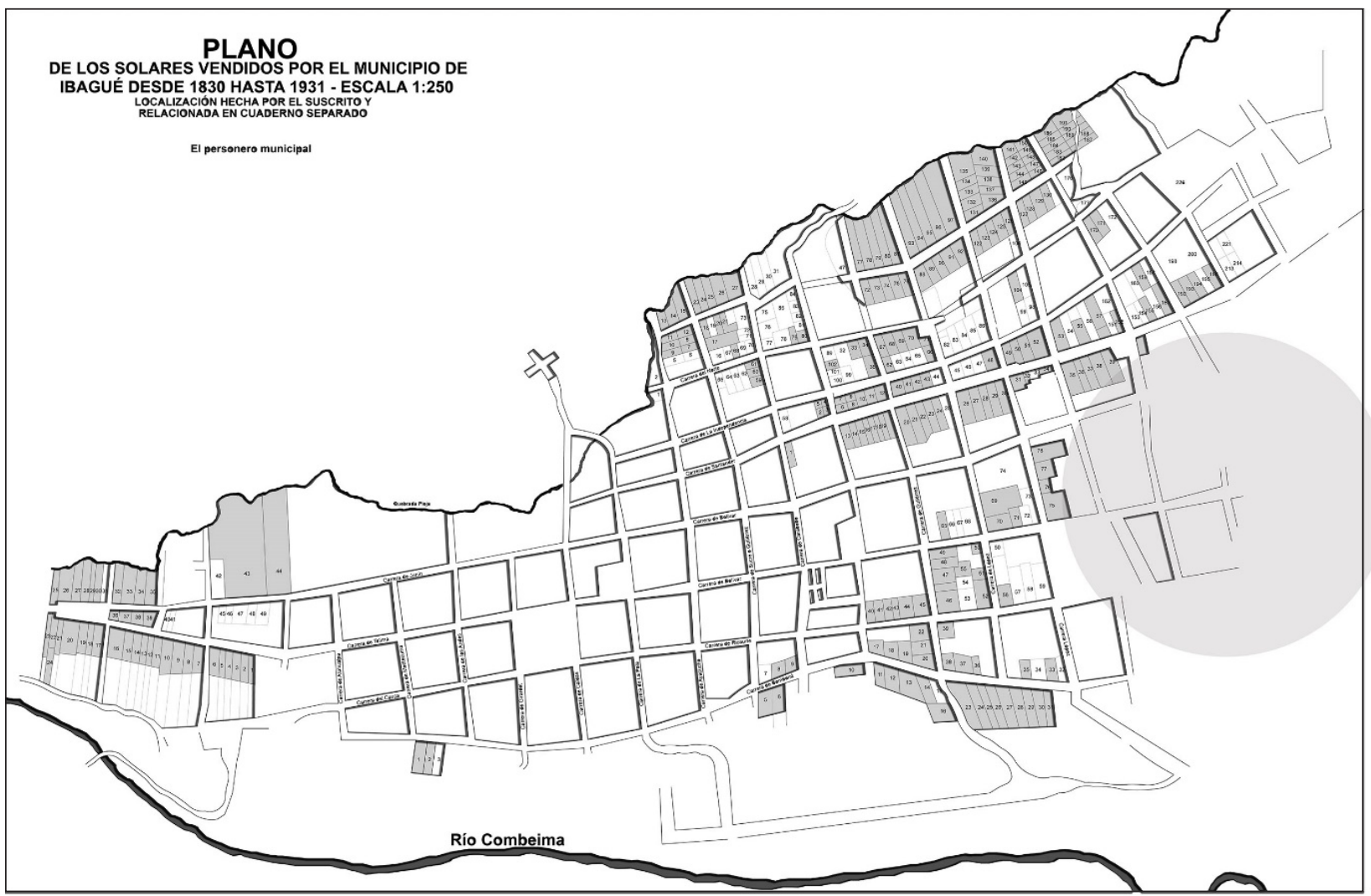

Digitalizado por Andrés Francel. Grupo de investigación e.arc. Facultad de Tecnologías. Universidad del Tolima, 2015.

Figura 4 - Plano de 1931 con la división predial y la zona de expansión de la calle 19 resaltada en el círculo Fuente: Elaboración propia (2015).

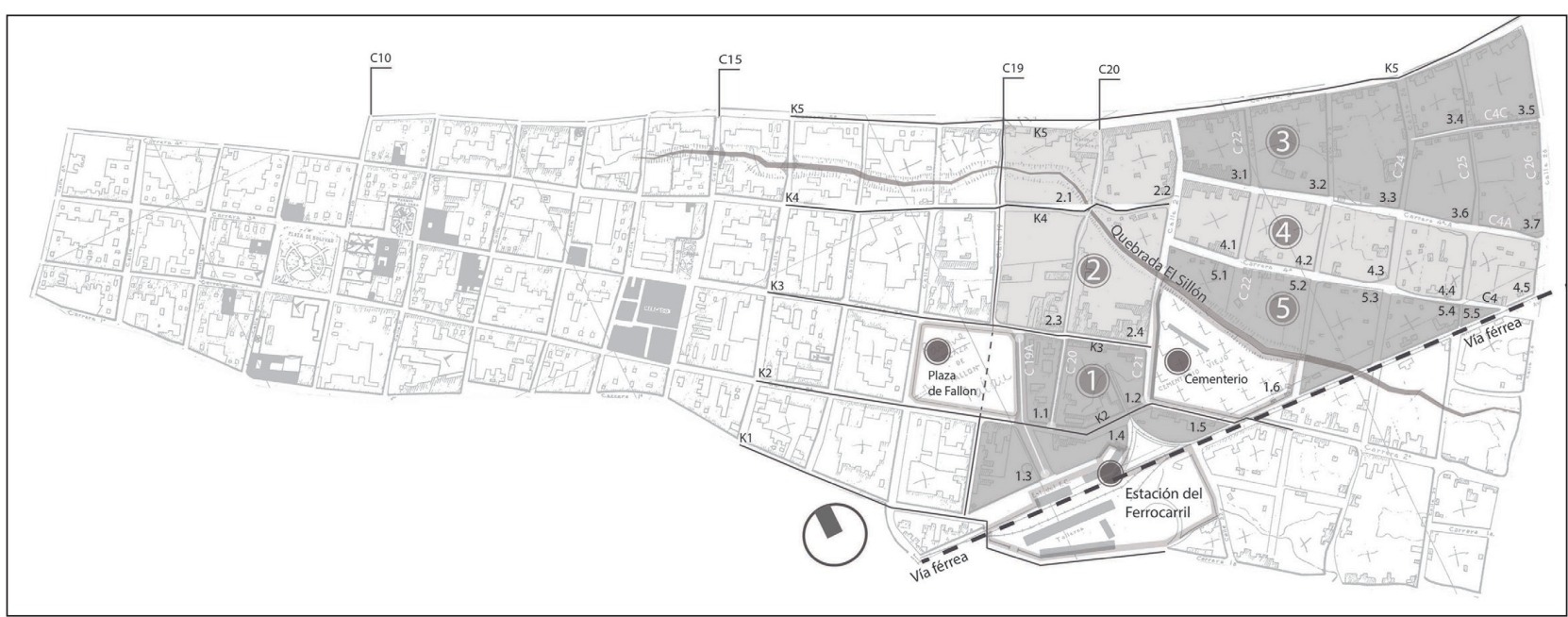

Figura 5 - Zonas de estudio localizadas en el plano de 1942. Elaboración propia (2016) sobre plano del IGAC (1942) Fuente: Biblioteca Darío Echandía del Banco de la República. 
(Figura 5). La numeración de los sectores se realizó en relación con la identificación de la calle 19 como eje hasta cuyo límite occidental se prolongó el trazado colonial y desde el cual se desarrolló el heteromorfismo hacia el oriente.
El primer sector y el fin de la ciudad colonial

El principal factor de fragmentación de la retícula urbana fue la plaza de Fallon porque interrumpió la calle 19 (entre las manzanas 1.3 y 2.3) y generó

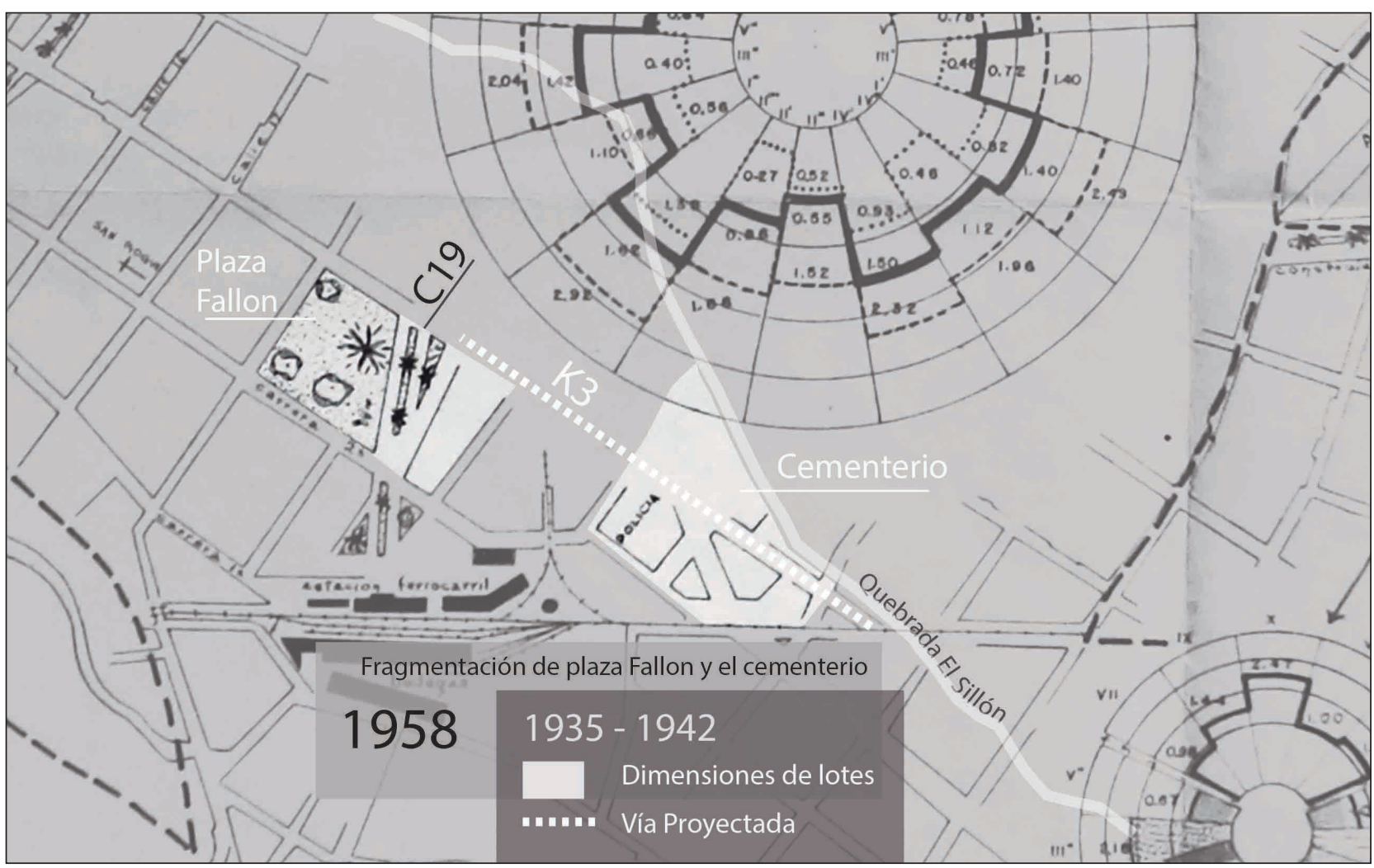

Figura 6 - Parcelación de la plaza Fallon y del cementerio entre 1935 y 1958. Elaboración propia (2016) sobre el plano de "Diagrama de niveles de vida" del IGAC, dibujado por Mireya Pérez B. (1958)

Fuente: Archivo Histórico Municipal.
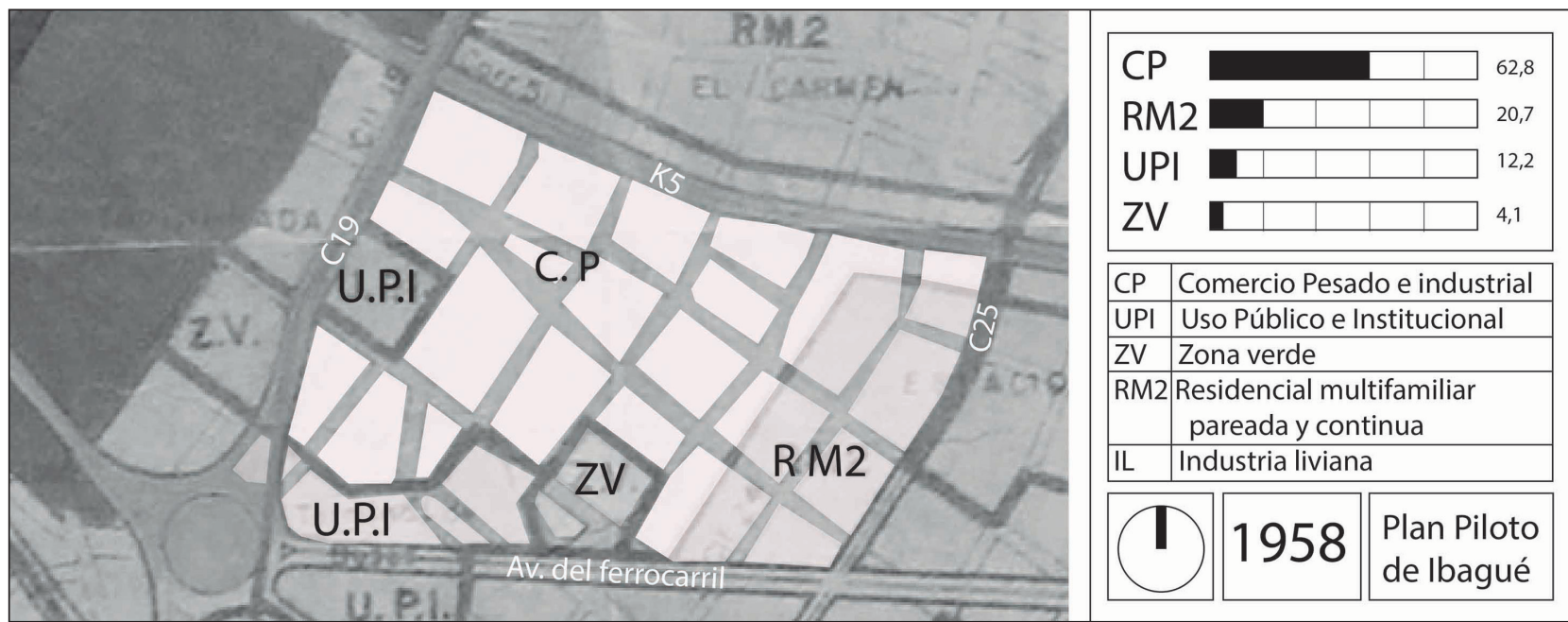

Figura 7 - Detalle del Plan Piloto de Ibagué (1966). Elaboración propia (2016) sobre Digitalización de Felipe Peralta (2015) Fuente: Archivo Histórico Municipal de lbagué. 


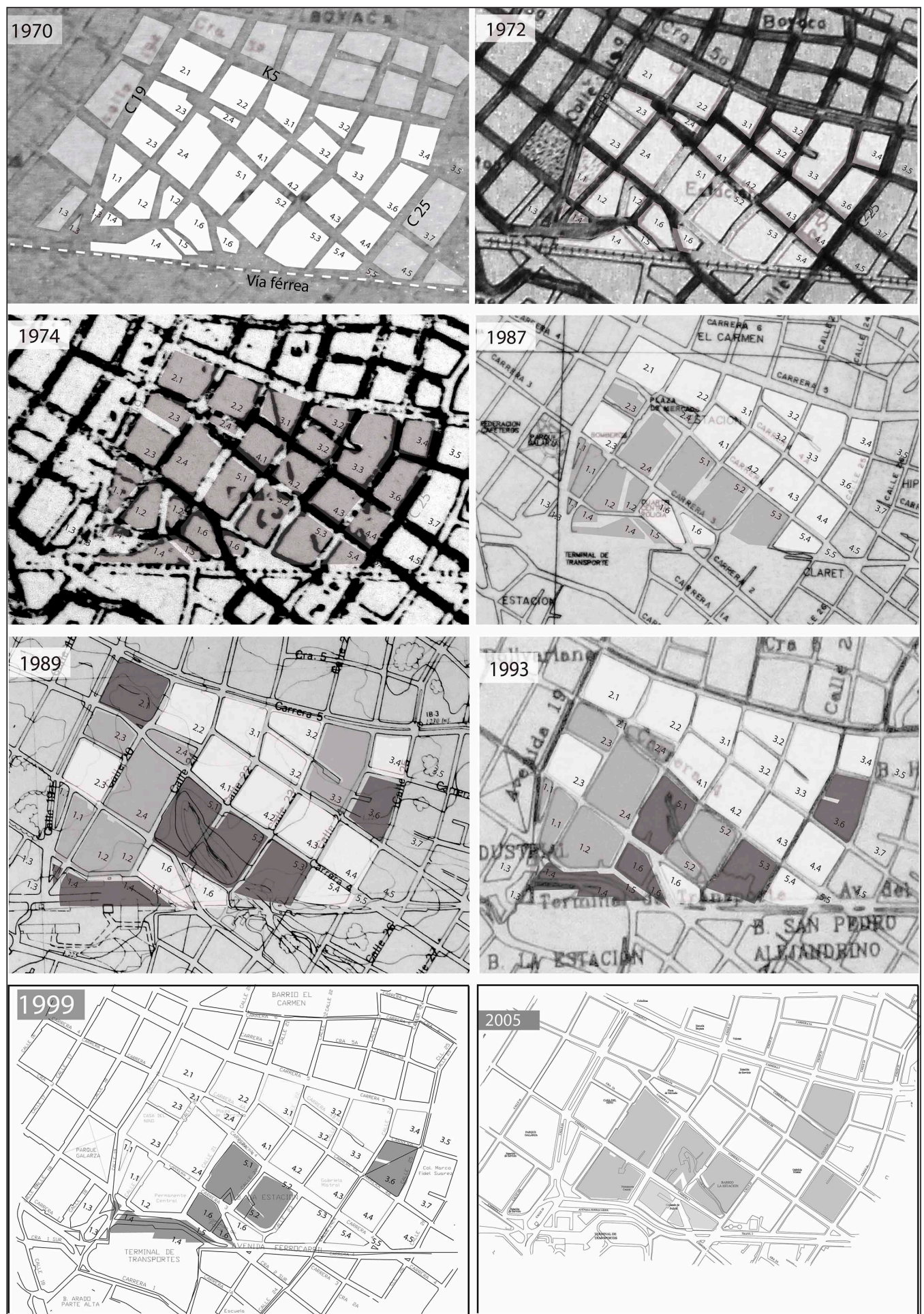

Figura 8 - Planos de evolución del sector. Elaboración propia (2016) sobre: Plano de la ciudad de Ibagué 1970. Departamento de planeación municipal. Sección técnica de dibujo. Dibujo de Juan J. Nieto P. Digitalización de Juan Ávila y Carlos Suárez (2015); Plano de la ciudad de Ibagué 1972. S.a. s.f. Digitalización de Juan Ávila y Carlos Suárez (2015); Plano de la ciudad de lbagué 1974. Fuera del marco se lee "Zonificación para el censo de la ciudad - Cámara de Comercio de lbagué - 1977. Digitalización de Juan Ávila y Carlos Suárez (2015); Plan de desarrollo e inversiones municipales de Ibagué 1987. Asociación para el desarrollo del Tolima (Alcaldía, FONADE). Dibujo de Noriega Restrepo \& Asociados Ltda. Digitalización de Juan Ávila y Carlos Suárez (2015); Plano aerofotogramétrico de la ciudad de Ibagué 1989. Ministerio de Hacienda y crédito público. Instituto Geográfico Agustín Codazzi. Digitalización de Juan Ávila y Carlos Suárez (2015); Plano de la ciudad de Ibagué 1993. Secretaría de planeación municipal. A partir de planchas acualizadas por el DANE según el censo. Digitalización de Juan Ávila y Carlos Suárez (2015) Fuente: Biblioteca Darío Echandía del Banco de la República. 
una nueva y corta calle 19A entre la manzana 2.3 y la estación Ospina. En el desplazamiento de la calle 19 y la generación de la 19A se observa por primera vez en el trazado urbano la preponderancia de las dimensiones de un espacio público sobre las cuadras circundantes. En los casos de fragmentación de las manzanas anteriores a 1942, las plazuelas generaron caminos internos a la manzana y se continuaron las vías existentes (Figura 9).

La interrupción vial continuó en las calles 20 y 21 que finalizaron en la carrera Segunda, contrario a la homogeneidad colonial, de modo que una anomalía se convirtió en repetición. La carrera Segunda fue inclinada hacia el norte entre las calles 20 y 21 (en el borde inferior de la manzana 1.2) y al llegar al cementerio (en la esquina inferior derecha de la manzana 1.2), retoma la dirección del trazado colonial y genera una arista entre las calles 22 y 23 (en el extremo derecho de la manzana 1.5) (Figura 5).

La inclinación de la Carrera Segunda fue consecuencia del segundo factor: la vía férrea. De hecho, la retícula urbana solo adoptó la nueva direccionalidad de la ferrovía en una parte de la cuadra 1.2. Esta característica presenta con claridad la superposición del paradigma nacional de desarrollo industrial e infraestructural (Arango, 1989) sobre el desarrollo urbanístico municipal que careció de diseños para armonizar el impacto de las ferrovías a pesar de las facilidades existentes a principios del siglo XX, como la propiedad gubernamental del suelo y la escasa construcción en la zona de expansión. Como consecuencia, a ambos costados de la vía se redujeron las dimensiones de las cuadras, se transformaron triangularmente y los ejes de las calles se desplazaron al norte y al sur de la vía férrea, acentuando la interrupción del trazado urbano (Figura 5).

El tercer factor para el heteromorfismo fue el cementerio, que no fue incluido en la cartografía de la década de 1930 porque se encontraba distante de las zonas de crecimiento urbano (Figura 4). Sin embargo, la proyección de las calles coloniales finalizó en el borde occidental del cementerio, marcado por la calle 21 (Figura 5). Allí se truncaron las carreras Tercera y Cuarta, la carrera Segunda fue desviada y, definitivamente, la carrera Primera quedó aislada de la malla urbana. El fin de la carrera Tercera representa la extinción del sistema urbanístico colonial porque a su alrededor se había desarrollado la ciudad hispánica, sus espacios públicos y los principales edificios (Figura 9). Así que literal y simbólicamente el cementerio fue el lugar en el que murió la ciudad colonial.

\section{Sectores 2 a 5}

En el segundo sector presenta la prolongación de las vías coloniales con un trazado irregular derivado de la reciente y parcial ocupación de los predios.

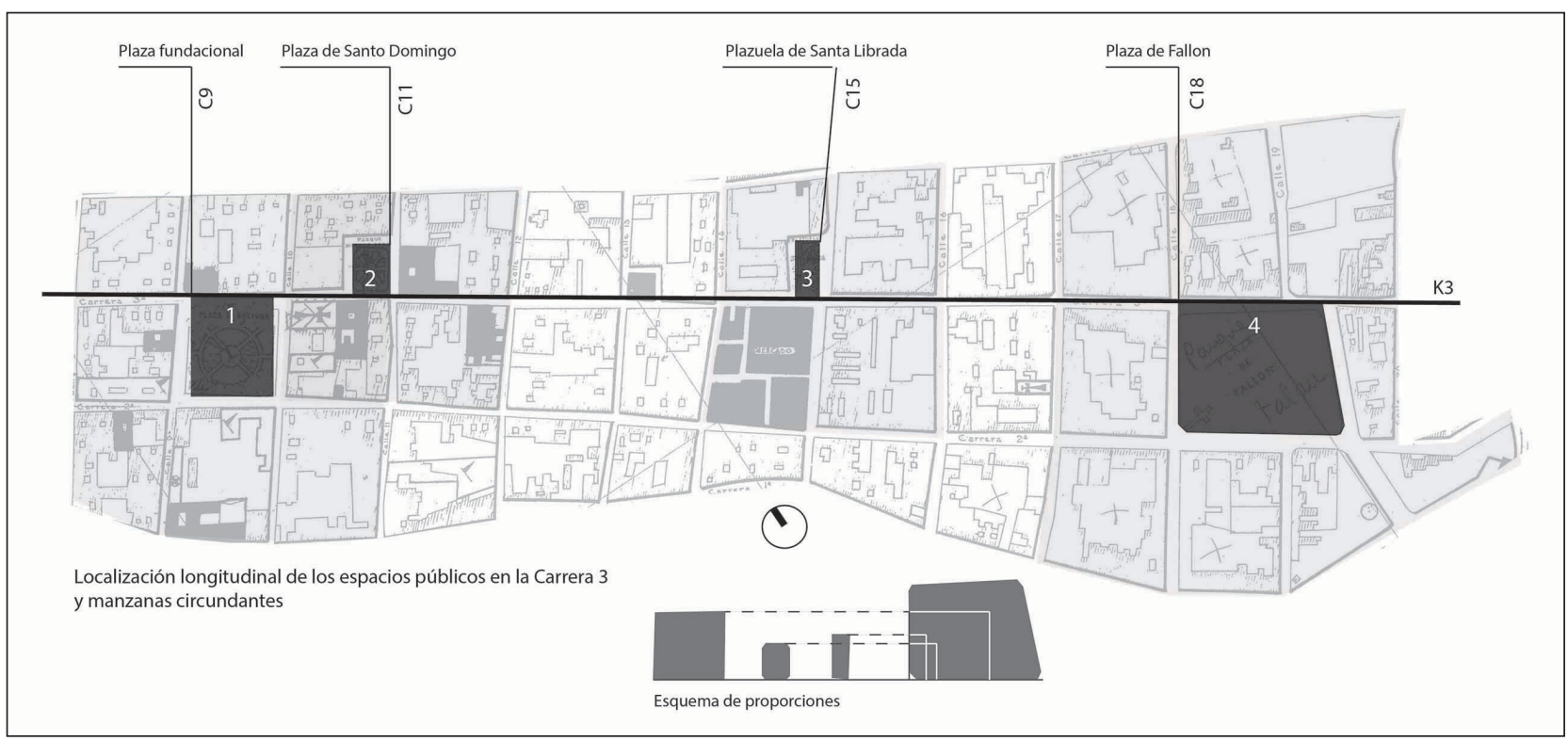

Figura 9 - Localización y proporción de los espacios públicos en 1942 Fuente: Elaboración propia (2016) sobre plano del IGAC (1942). 
Se observa crecimiento triangular en las manzanas $2.3 \mathrm{y}$ 2.4 entre las carreras Tercera y Cuarta, lo cual generó cuadras con el doble del tamaño de las coloniales. Aparece la quebrada El Sillón como primer elemento urbano anómalo entre las manzanas 2.1 y 2.4, lo cual evidencia la indiferencia de la retícula con respecto a la topografía (Figura 5).

El tercer sector, ubicado al oriente de la calle 21, presenta la desconexión con las vías de la ciudad colonial. Su crecimiento es triangular y repite la generación de nuevas vías discontinuas como la calle 22 (que termina en el cementerio) y la carrera Cuarta $\mathrm{C}$ que comienza en la calle 24 (entre las manzanas 3.4 y 3.6). El cuarto sector desarrolla una franja de manzanas de dimensiones homogéneas entre dos nuevas carreras discontinuas, la Cuarta y la Cuarta A con orientación paralela a la traza colonial pero dislocada de esta en la calle 21 . La manzana 4.5 genera aristas residuales como constante de relación entre la retícula urbana y la vía férrea.

El quinto sector se configuró entre las diagonales de la quebrada El Sillón, la vía férrea y el eje desconector de la calle 21, por lo cual su forma general es triangular y ninguna de sus manzanas presenta regularidad. Como consecuencia, la manzana 5.5 se configuró como una zona residual o demarcación con "dispersión de tipologías, dimensiones y categorías" (Cirer-Costa, 2011, p. 12) cuyas dimensiones impiden estructuraciones de utilidad y coherencia con el entorno (Sabaneta, 2009 , p. 131) y que generalmente son resultado de la prioridad del tránsito vehicular (Franco Taipe, 2014, p. 11-12).

La vía férrea generó zonas residuales longitudinales a su trazado, por lo cual fue clasificada como una cicatriz urbana (Xolalpa, 2015). Debido a que este término es de uso común, pero carece de especificaciones, se tomó la definición médica (Sánchez-Mateos García et al., 2015) y se apropió al ámbito urbanístico, proponiendo que una cicatriz es una alteración permanente de la superficie urbana como consecuencia de un daño y se constituye en testimonio de sus reparaciones.

El resultado del análisis de los sectores es que el cambio morfológico (heteromorfismo) respecto de la ciudad colonial, corresponde con un cambio conceptual urbanístico centrado en el desarrollo económico cuyo símbolo es el ferrocarril. El comercio inmobiliario alrededor de la estación rompió el paradigma de crecimiento urbano homogéneo e instaló como criterio urbanístico el libre mercado inmobiliario debido a la urgencia de recaudo del gobierno municipal para reinvertir en obras de infraestructura.

\section{Las propuestas de costura urbana en 1935}

La desarticulación entre la zona estudiada y el resto de la ciudad fue evidente desde 1935, motivo por el cual se proyectó un modelo de ciudad diferente para "coser" la herida urbana (Franco Taipe, 2014) creada por las dinámicas del ferrocarril. Para controlar la expansión de la ciudad se generó el plan urbanístico Ibagué Futuro de 1935 por la Sociedad Industrial de Ingeniería y Comercio (SIDEICO). Su origen se encuentra en la labor de Ricardo Olano como promotor urbanístico, quien generó los congresos nacionales de urbanismo $(1917,1921)$ y el diseño de los planes Medellín Futuro (1907-1913), Bogotá Futuro (1923-1925) y la aprobación de planos para el desarrollo de las ciudades colombianas con más de 10.000 habitantes (Alba Castro, 2013), proyecto dentro del cual se encuentra Ibagué Futuro. Sus estrategias pueden sintetizarse en los ocho proyectos que se describen a continuación (Figura 10):

1) La proyección de la Avenida Quinta era la formalización del camino que conducía a Bogotá desde tiempos coloniales. Se planeó con doble calzada, zonas complementarias como parqueaderos, parques y se prohibió el desarrollo inmobiliario en su límite sur, tomando en cuenta que la abrupta topografía de la cuenca de la quebrada El Sillón implicaría mayores costos de construcción (Figura 11).

2) En lugar de los desarrollos inmobiliarios, se planteó la generación de bosques para aprovechar las características del suelo y la topografía de la quebrada. Se dotaba a la ciudad de un espacio público de grandes dimensiones del cual carecía y requería en el futuro. El bosque se generaba en la pendiente más fuerte del terreno para estabilizar el suelo en una franja cuyo ancho iba desde 120 hasta 300 metros (Figura 12).

3) Los jardines del Sillón se ubicaban en la zona con menor inclinación de la ribera de la quebrada hacia la actual carrera Cuarta (Figura 12). De este modo, la traza colonial quedaría completamente suspendida entre las calles 19 y 24, la Carrera Quinta y la Avenida del Café y se creaba la zona verde de articulación entre la ciudad antigua y la 


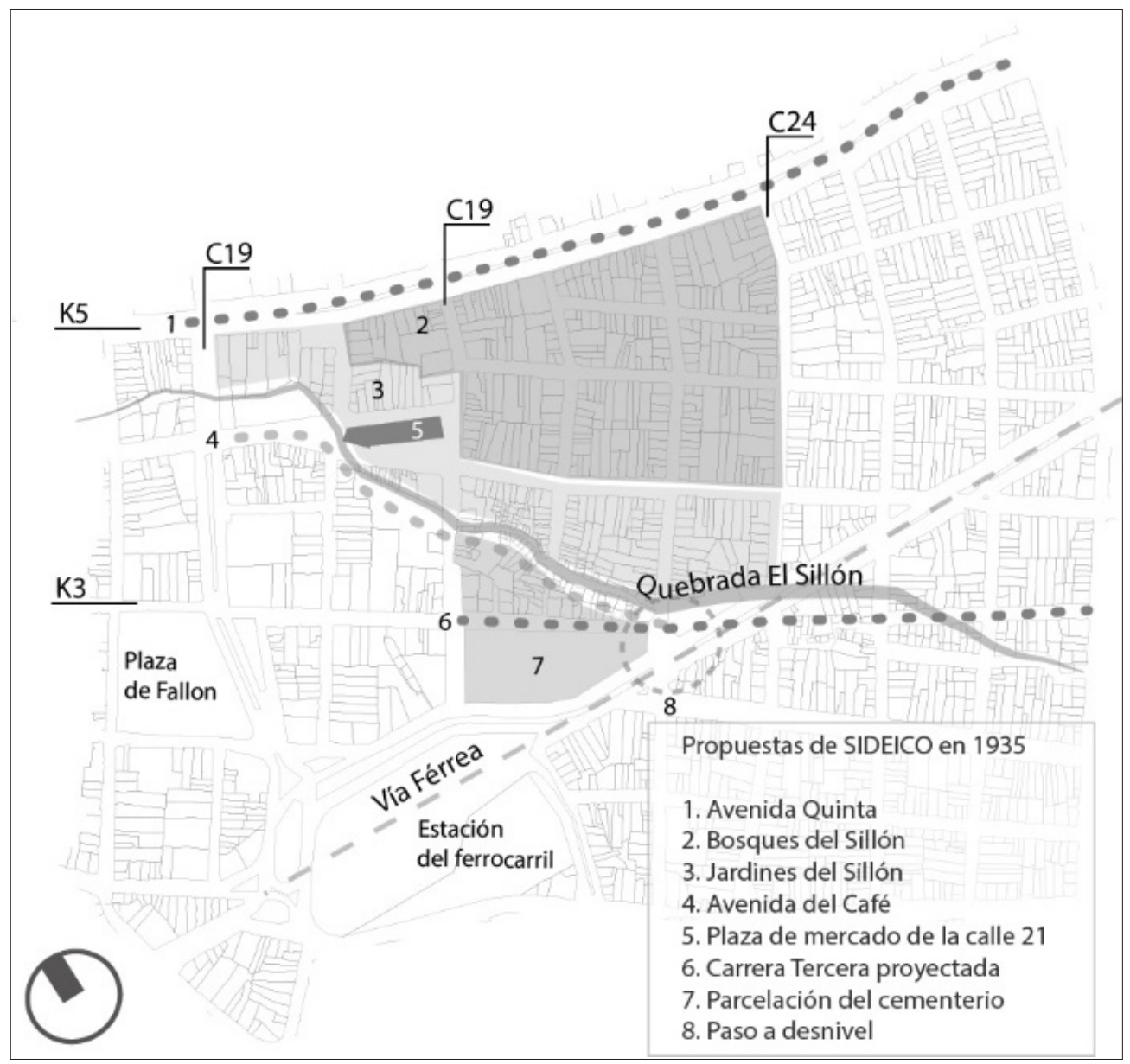

Figura 10 - Propuestas de SIDEICO en 1935

Fuente: Elaboración propia (2016) sobre plano del Plan de Ordenamiento Territorial de lbagué (2014).

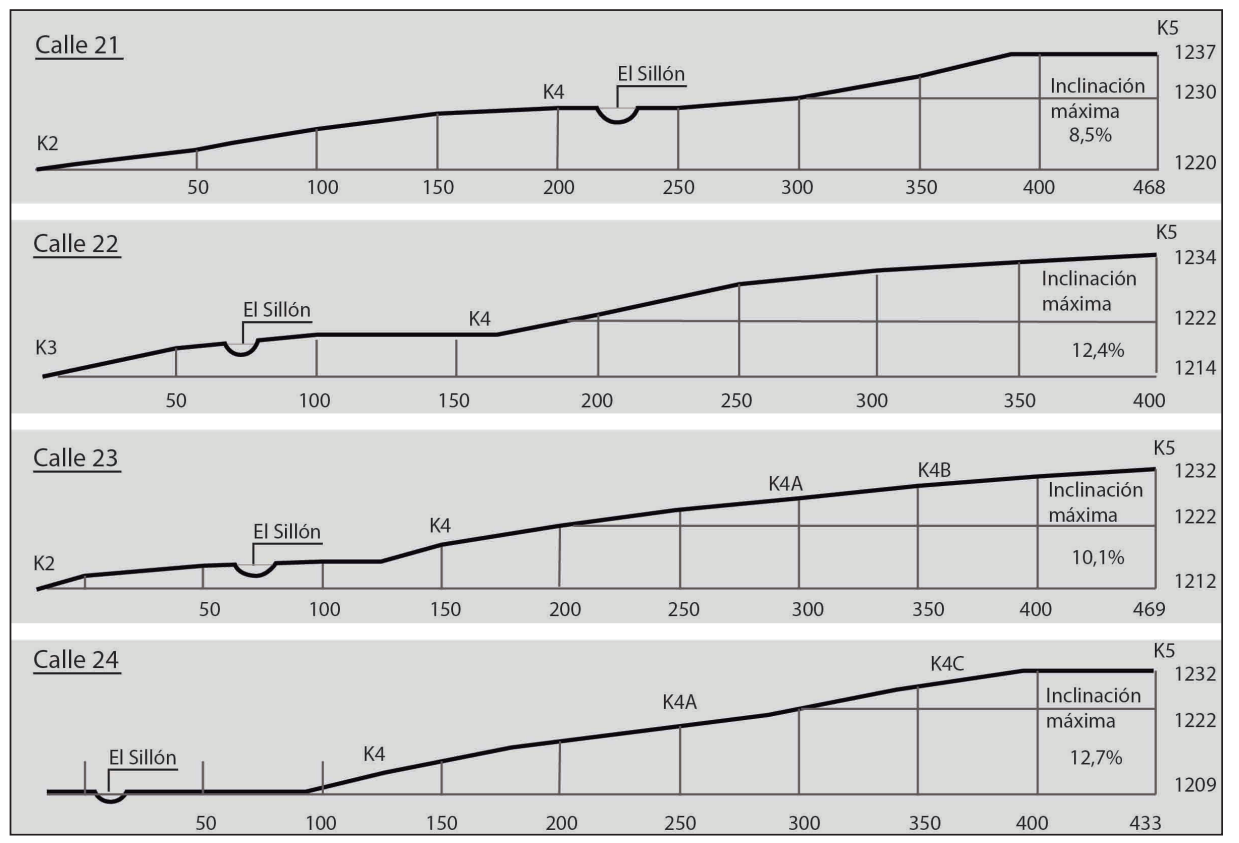

Figura 11 - Perfiles de elevación de las calles

Fuente: Elaboración propia (2016) a partir de los datos de Google Earth. 


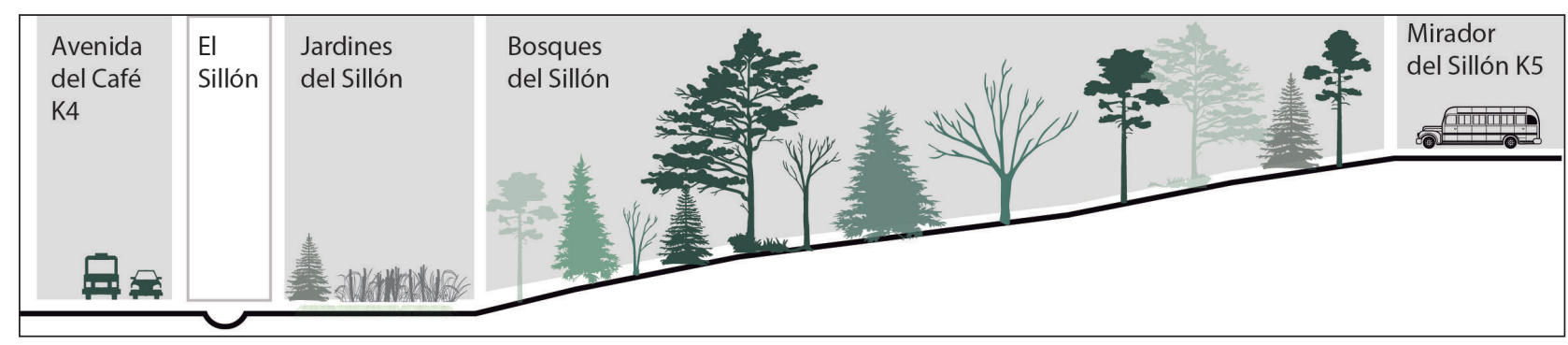

Figura 12 - Paisaje del Sillón planeado en 1935

Fuente: Elaboración propia (2016).

ciudad futura cuyo corazón era una fuente hídrica para adaptarse a los paradigmas funcionalistas de 1935.

4) La avenida del Café se planeó con el propósito de dar continuidad a la carrera Cuarta pero rompiendo el trazado colonial, de manera que se protegía el curso natural de la quebrada El Sillón y se generaba un nuevo modelo de desarrollo de ciudad en el que el trazado urbano correspondía con la topografía. La Avenida del Café es el corazón de la propuesta para el desarrollo del sector estudiado porque rompe con el trazado colonial reticular y decide tomar como guía para su trazado el lecho de la quebrada (Figura 10), con lo cual se logra subsanar la desarticulación fundamental entre la planeación urbana y la topografía procedente de tiempos coloniales. Esta avenida generaba una intersección con la vía férrea en la calle 23 , donde se planteó un paso a desnivel.

5) Para generar coherencia en el desarrollo urbanístico de esta zona y evitar que se poblara espontáneamente, se planteó la construcción de la plaza de la 21, la cual se convertiría en el elemento organizador de los flujos comerciales y de la infraestructura complementaria para bodegaje y comercios mayoristas y minoristas. Así se evitaba que las actividades residenciales fueran afectadas por el desarrollo comercial de la plaza de mercado y se generaba un gran espacio público de transición entre los usos.

6) La proyección de la carrera Tercera desde la calle 21 hasta la vía férrea tenía como propósito la proyección futura de la ciudad a ambos costados de la vía férrea. Su ejecución requería de las dos propuestas siguientes.

7) El traslado del cementerio y su parcelación (Figura 13) para permitir la proyección de la carrera Tercera, idea que inicialmente no fue contemplada, como se afirma en el informe, pero que después fue considerada como fundamental. En este cambio de decisiones se observa el inicio del problema inmobiliario que aconteció posteriormente en la zona, pues el principio de convertirla en una franja verde de amortiguación entre la ciudad colonial, el mercado y la ciudad funcional fue desplazado por la parcelación del cementerio.

8) La generación de pasos vehiculares y peatonales a desnivel fueron los puntos de costura que visualizaron los planeadores de 1935 para conectar las márgenes de ciudad a ambos lados de la vía férrea. De esta propuesta de pasos a desnivel se deduce que SIDEICO identificó claramente el problema de ruptura de la ciudad en dos márgenes situadas a ambos lados de las líneas del ferrocarril, la necesidad de coserlas y la imposibilidad de lograr una armonía urbana entre ellas.

La proyección de ciudad desde el análisis gráfico del movimiento Economía y Humanismo de 1958

En el plano titulado "Diagrama de niveles de vida" de 1958, se observa una pequeña parte de la zona estudiada, debido a que la gráfica de datos fue superpuesta al trazado urbano (Figura 6). Sin embargo, la existencia de este plano es reveladora para la historia de la ciudad y de la zona porque es testimonio de la influencia del movimiento Economía y Humanismo, asociación francesa fundada por Louis-Joseph Lebret en 1941 en Marsella, cuyo propósito fue la promoción de "[...] la economía al servicio del bien humano, en una adaptación al contexto social" (Leite Trindade \& Dornelas Câmara, 2014, p. 5). 


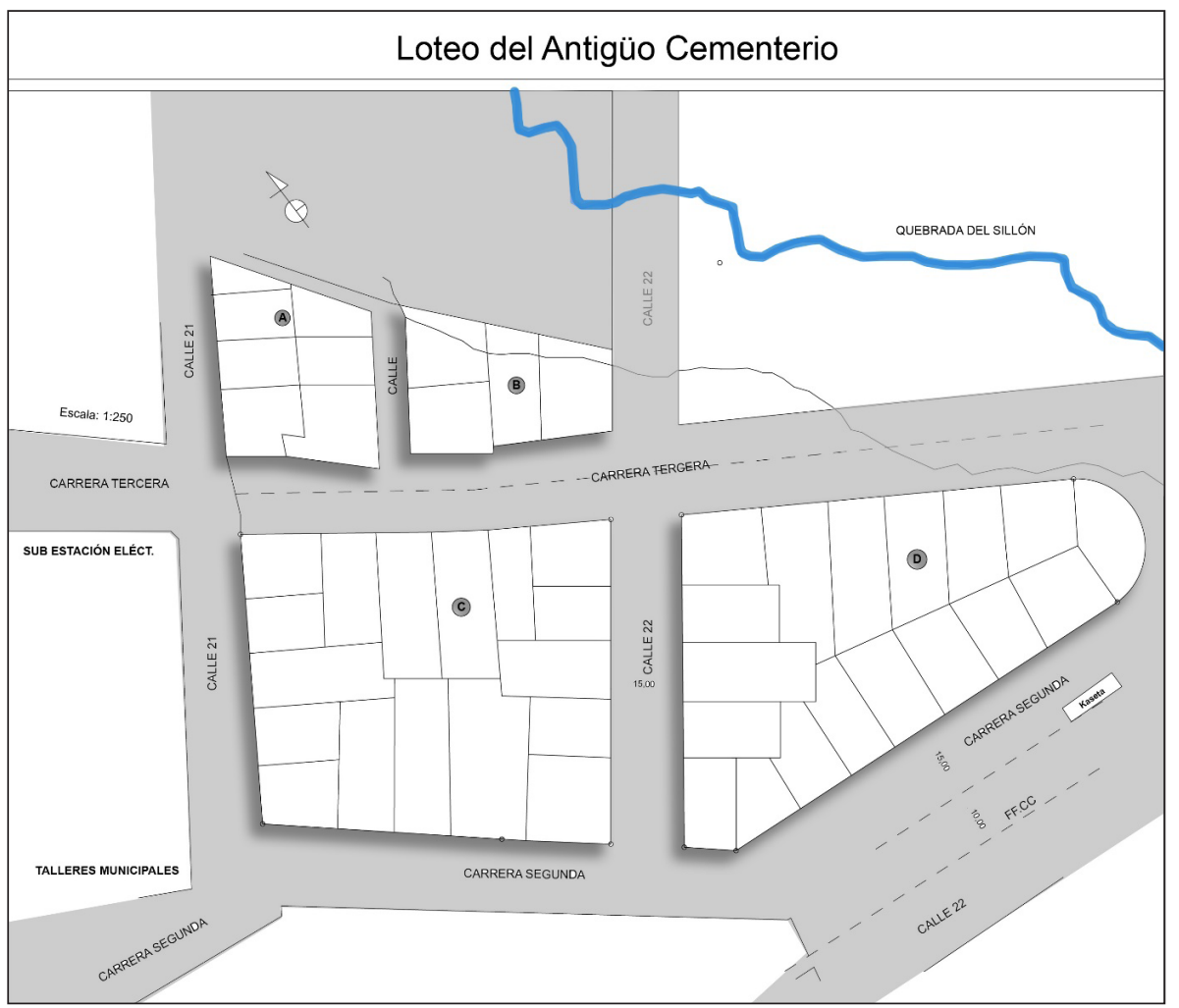

Figura 13 - Loteo de los terrenos del antiguo cementerio. Vectorización propia (2015) del original (s.f.)

Fuente: Archivo Histórico Municipal de Ibagué.

Los principios de la economía humanista arribaron institucionalmente a Colombia en 1955 mediante la figura de la Misión Lebret, convocada por el Comité Nacional de Planeación con el propósito diagnosticar el nivel de vida de la población, contar con perspectivas de la situación económica nacional, adelantar una planeación racional y determinar las necesidades educativas (Arévalo Hernández, 1997, p. 10). La captación de datos mediante encuestas con orientación sociológica se realizó entre el primero de febrero y el 30 de septiembre de 1955 (Bogotá, 1958, p. 55). El estudio entregado abordó aspectos fundamentales para la comprensión de las 11 principales ciudades colombianas, la mayoría de las cuales fueron capitales de Departamento.

El diagrama de mayores dimensiones se encuentra sobre la zona estudiada, lo cual impide una revisión total de su morfología. Las gráficas se ubicaron sobre los sectores con menor desarrollo económico humanístico, lo cual significa que la zona de estudio era percibida en 1958 como un lugar con bajo nivel de vida (Figura 14). Sin embargo, el sector analizado presenta el comportamiento más homogéneo y mayor índice de desarrollo entre los diagramados, por lo cual los datos para su análisis son más predecibles, mientras que en las demás zonas, las medidas medias son muy bajas y su predicción es mínima (Juaristi Linacero, 1984). Los demás sectores diagramados eran periféricos, mientras que el espacio del Sillón se ubicaba en el centro geométrico de la ciudad, lo cual indica que era el sector más poblado y degradado en sus alrededores (Figura 14 y 15).

En el gráfico es claro que todos los niveles de vida analizados en el sector presentan condiciones entre "desastrosas y malas" según los criterios de la misión. En lo respectivo a lo urbanístico y arquitectónico, las medidas correspondientes fueron 0,72 y 1,12, lo cual es alarmante pero coherente con la observación de la misión en cuanto a una medida general del $50 \%$ de la población nacional en la miseria. De ello se puede deducir que la conjunción de las dinámicas de comercio alrededor de la estación del ferrocarril, el mercado inmobiliario en el sector y la falta de implementación de los criterios generados en 1935 por el plan Ibagué Futuro, degradaron sistemáticamente la zona de la calle 21. 
En cuanto al análisis comparativo del sector en 1958 con respecto a las características examinadas en 1935 y 1942, se evidencia la parcelación del cementerio propuesta por SIDEICO, el trazado de la carrera Tercera y la recomposición de la calle 19 que reconfiguró la plaza Fallon y legó a la ciudad una zona compuesta por numerosos y heteromórficos residuos urbanos (Figura 6). Claramente, no se aplicaron las

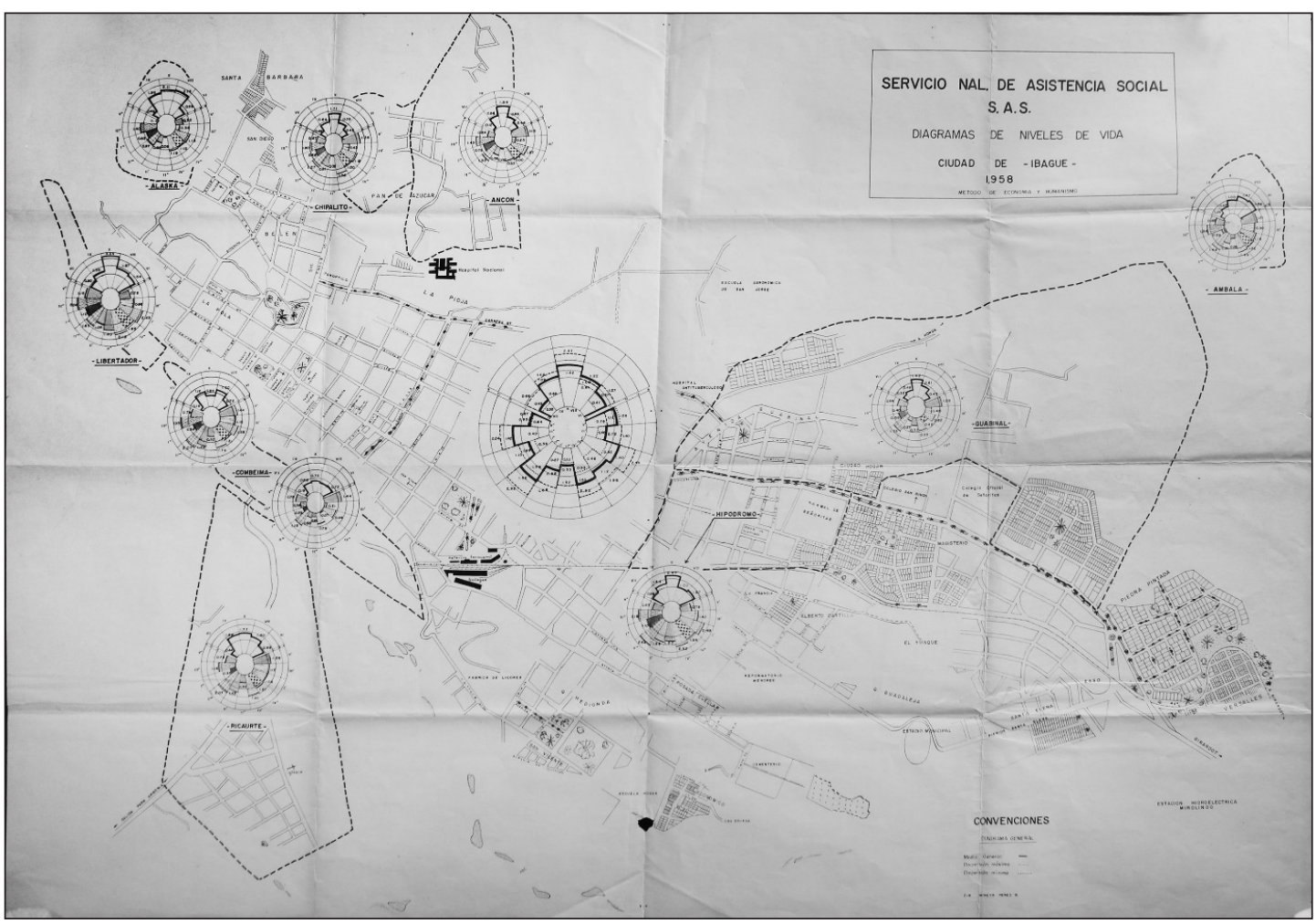

Figura 14 - Diagrama de niveles de vida del Servicio Nacional Asistencial. Dibujo de Mireya Pérez B. (1958). Digitalización de Felipe Peralta (2015) Fuente: Archivo Histórico Municipal de Ibagué.

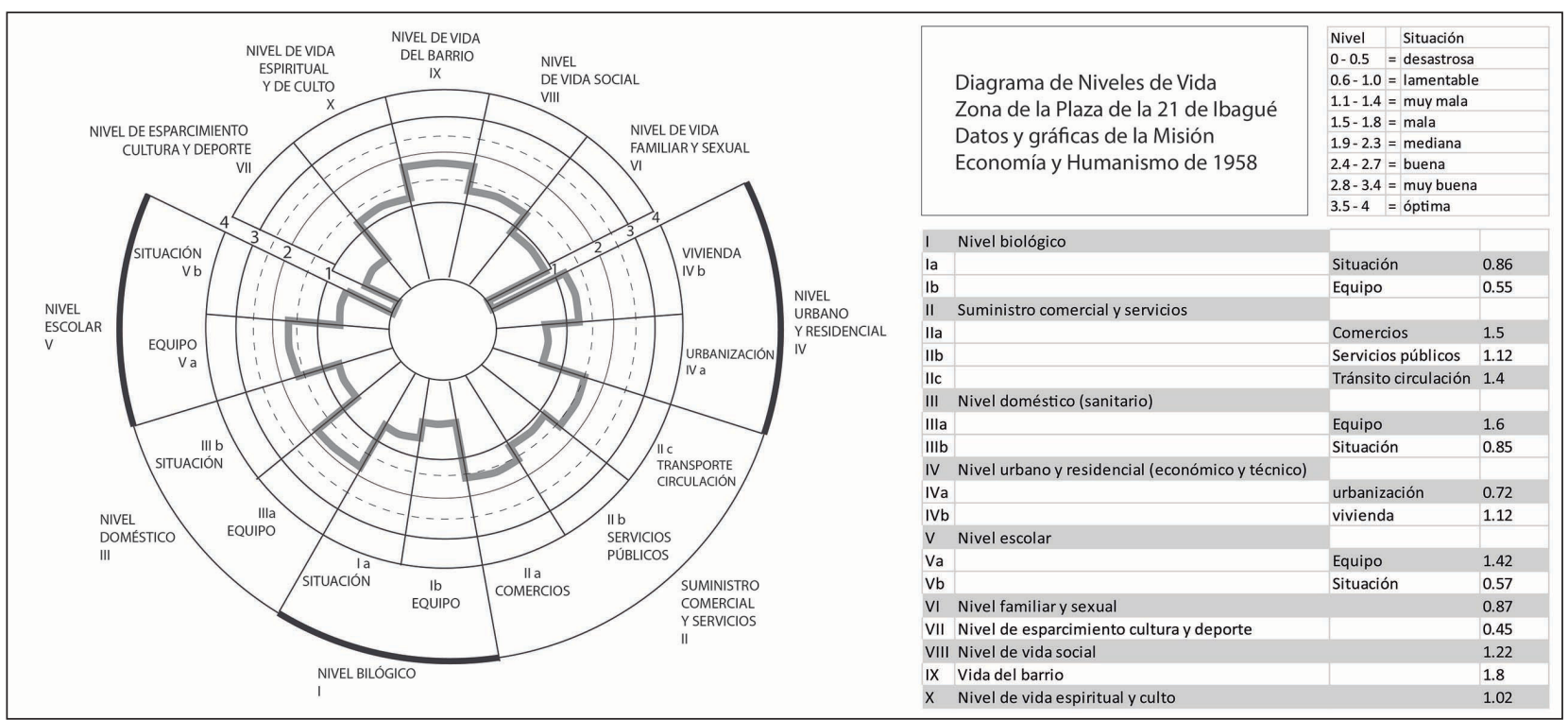

Figura 15 - Diagrama de niveles de vida en el sector de la calle 21

Fuente: Elaboración propia (2016) a partir de los datos de la Misión Economía y Humanismo (1958). 
costuras urbanas propuestas en 1935, la vía ferroviaria continuó como divisor sectorial y la trama urbana se modificó irreversiblemente con nuevos modelos barriales, como se observa en la margen derecha de la Figura 15.

\section{El Plan Piloto de 1966}

El siguiente plano en el que se encuentra el sector de la Calle 21 es el Plan Piloto de 1966, en el cual se observa la implementación de los instrumentos de planeación del movimiento moderno consignados por Le Corbusier, Sert y Wiener en el Plan para Bogotá en 1953, como el énfasis organizativo a partir de los centros cívicos urbanos y barriales, la jerarquización de los siete tipos de vías desde las nacionales hasta las peatonales y la representación gráfica como modelo de análisis y de síntesis (Tarchópulos, 2006). Sin embargo, las críticas al plan para Bogotá y la crisis mundial del movimiento moderno generaron una vertiente de planeamiento urbano que pasó del enfoque funcionalista con validez universal a uno de base social con adaptación a los entornos (Ramírez Ríos, 2011, p. 42-45). Esto significa que se pasó de cumplir las "funciones" de residir, trabajar, movilizarse y recrearse, hacia la comprensión de las necesidades de asociación, identidad, patrones de crecimiento, movilidad y cadenas productivas (Ramírez Ríos, 2011, p. 45).

Como consecuencia, el plan de 1966 estuvo enfocado en la clasificación de 19 zonas de desarrollo que vinculaban las dinámicas geográficas (hídricas, forestales, orográficas), sociales (residenciales, recreativas) y las económicas (agronómicas, comerciales e industriales). Su soporte se encontraba en la estructura vial cuya meta fue la continuidad y potenciación de las conexiones históricas entre Ibagué y el resto de país (Martinez Silva, 2016). Los principios para este desarrollo fueron trazados por los arquitectos Fernando Ruiz Gutiérrez, Carlos Martínez Silva y Jorge Peñaranda Ordóñez, funcionarios del Instituto Geográfico Agustín Codazzi (IGAC) en respuesta al crecimiento sostenido de la población nacional que en el caso de Ibagué presenta una variación del $230 \%$ entre sus 54.381 habitantes en 1951 y 129.930 en 1965 (ACEP, 1975, p. 30).

Es evidente en el plano que el sector está consolidado y no se propusieron intervenciones sobre las manzanas (Figura 7). El núcleo de la propuesta fue la primacía del automóvil sobre los medios de transporte anteriores, por lo cual la vía férrea en desuso fue propuesta como avenida del ferrocarril, en cuyo remate con la Calle 19 se planteó un rond-point o glorieta que se sumó a la fragmentación de la trama urbana con la consecuente generación de residuos urbanos.

La plaza de mercado de la calle 21 generó dinámicas comerciales "pesadas", por lo cual la vivienda fue desplazada al límite oriental del sector. Las zonas verdes fueron concentradas hacia el sur en colindancia con la avenida del ferrocarril y se implementó un centro de ayuda para la población en condiciones de extrema vulnerabilidad (UPI). La importancia de las relaciones comerciales entre las nuevas construcciones y el mercado condujeron a que las viviendas quedaran inmersas en un lugar comercial sin las transiciones necesarias para evitar conflictos entre los usos ni mejorar la calidad de vida residencial. Las zonas verdes quedaron distantes y desintegradas de las viviendas.

\section{Ciudad y superposición}

planimétrica desde 1970

Al comparar los cambios morfológicos entre 1970 y 2014, la numeración inicial permitió rastrear la fragmentación de las cuadras, por lo cual se observan varias formas urbanas con la misma numeración, principalmente las manzanas 1.1, 1.4, 1.6, 2.3, 3.2 y 5.2. Se asignaron intensidades a los rellenos de las manzanas, de modo que los tonos oscuros indican mayores cambios en comparación con el plano inmediatamente anterior (Figura 8).

Los planos de 1970, 1972 y 1974 conservan la morfología descrita en 1966 y muestran que la glorieta planteada en este último nunca se construyó. La vía férrea es clara hasta el plano de 1974, pero a partir de 1987 su trazado se desdibuja hasta transformarse en Avenida del Ferrocarril en 1993. En 1987 se generaron bahías de estacionamiento vehicular en las manzanas 1.1, 1.2, 2.3 y 2.4, se fragmentó la manzana 1.1, se fusionaron las manzanas 1.2 y el nuevo terminal de transportes englobó las manzanas 1.4 y 1.5. A partir de 1987 existe una constante fragmentación y agrupación de manzanas hacia el centro, suroccidente, nororiente del sector y a partir de 2005 se encuentran graficados los andenes. 
La relación entre las transformaciones y las intensidades permitió la identificación de patrones de interpretación para los fenómenos del sector. Se desarrollaron tres etapas, comenzando por la superposición de planos en orden cronológico mediante la generación de capas en Photoshop, de modo que el plano más antiguo (1970) quedó en el fondo y el de 2005 en la superficie. A cada capa se asignó una opacidad de 30\% y un relleno del $80 \%$ para permitir la observación general de cambios. A la imagen resultante se aplicó la segunda etapa, consistente en la manipulación de niveles de contraste para la facilitar la observación de cambios, a lo cual se denominó proceso de revelado en correspondencia con la técnica de fotografía análoga. La tercera etapa consistió en la duplicación de la imagen revelada y la generación de una máscara de recorte en Illustrator, en la cual se evidenciaron con máximo contraste los principales cambios acontecidos entre 1970 y 2014 (Figura 16).

La validación entre el plan de ordenamiento territorial y la superposición histórica

Como paso final se tomó el polígono de la máscara de recorte y se comparó con el Plan Parcial de la calle 19 (Ibagué, 2013), perteneciente al Plan de Ordenamiento
Territorial (POT) (Ibagué, 2002). Un POT consigna las disposiciones generales sobre el desarrollo de la ciudad y un plan parcial genera coherencias entre áreas urbanas específicas y el marco municipal de referencia (Ibagué, 2000).

El plan parcial de la calle 19 es una estrategia de renovación urbana común a 9 ciudades colombianas con una metodología de identificación, diseño y concertación entre los entes públicos, privados y la comunidad (Colombia, 2012). Por consiguiente, es el planteamiento urbanístico más reciente sobre el sector de estudio, coordinado directamente con el ámbito nacional. La última fase del análisis constó de la superposición entre el polígono delimitado por el plan parcial (Angulo Posse, 2011) y el hallado en la investigación sobre la transformación histórica (Figura 17).

En la superposición de polígonos es evidente la convergencia entre la zona central y la suroccidental, correspondiente a los números 1.3, 1.3, 2.1, 2.4, 2.4M (plaza de mercado), 5.1 y 5.2. Las manzanas 1.4 y 1.5 se encuentran fuera de la convergencia porque pertenecen al borde del terminal de transportes, el cual es incluido en el plan parcial, a pesar de estar fuera del polígono de delimitación, por lo cual se asimilan como parte de la convergencia histórica. Las demás cuadras que componen el plan parcial han tenido modificaciones desde 1931, por lo cual se

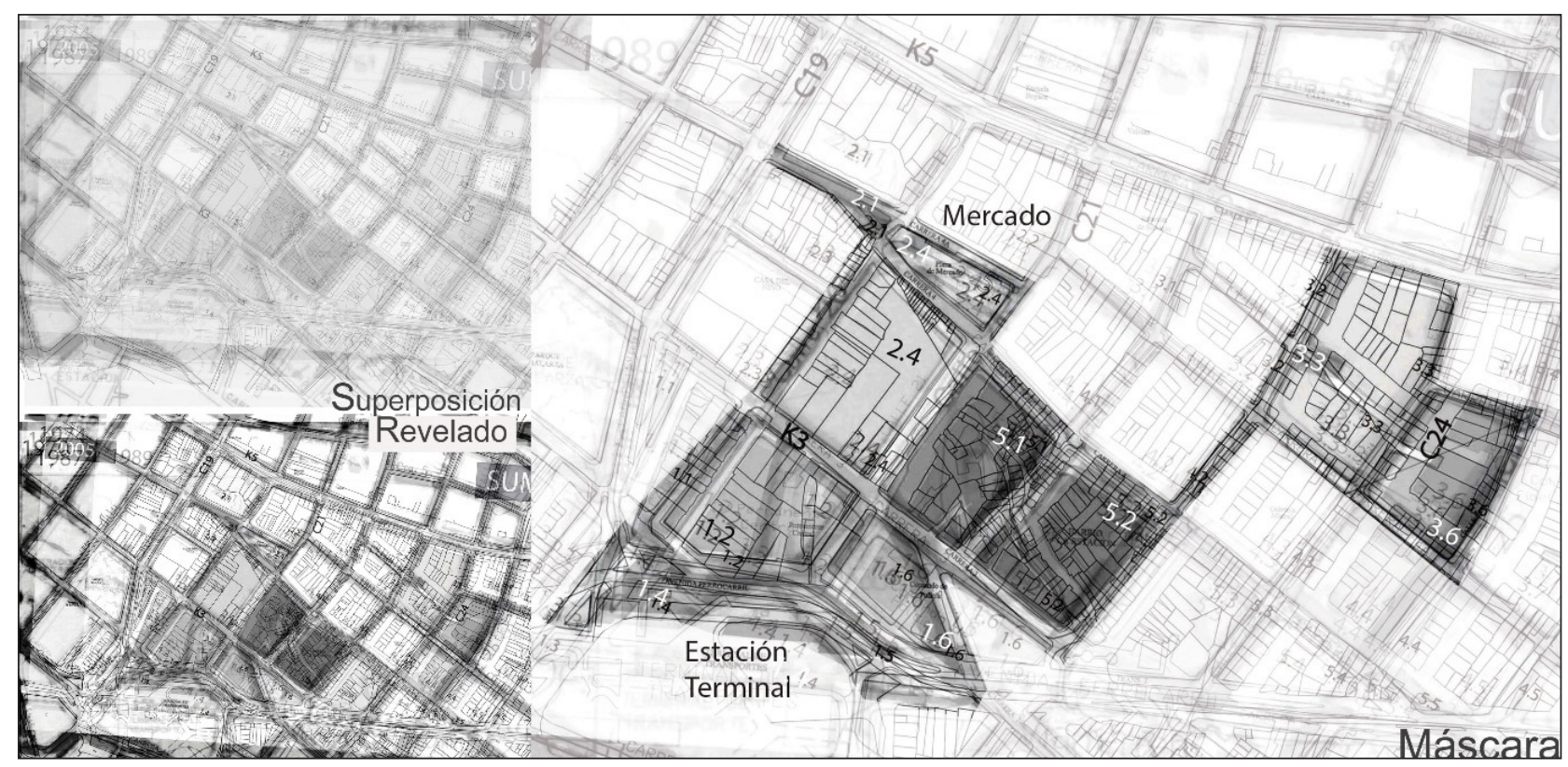

Figura 16 - Proceso de superposición, revelado y determinación del polígono histórico de cambios Fuente: Elaboración propia (2016). 


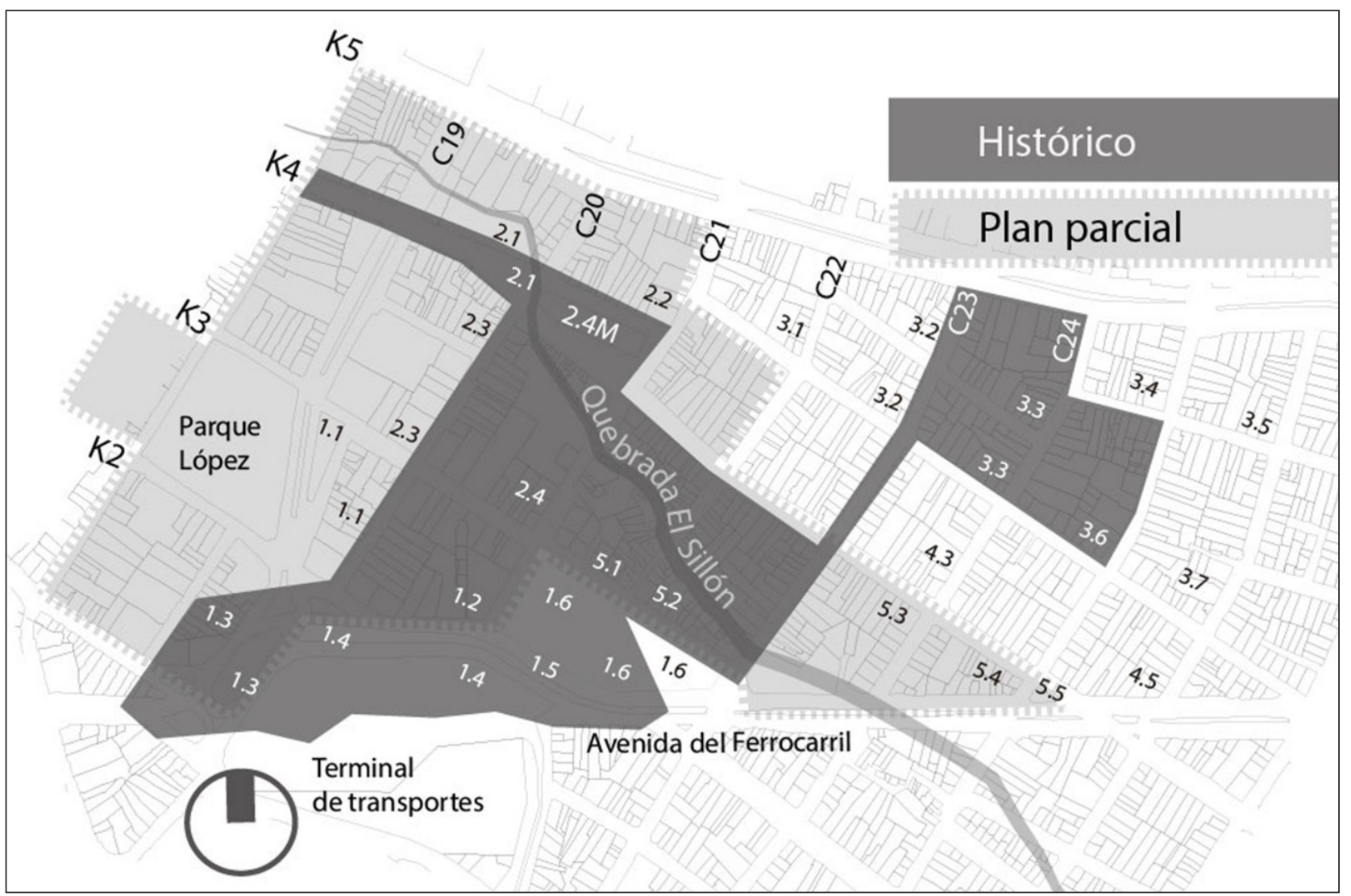

Figura 17 - Comparación entre los polígonos del Plan Parcial y del revelado histórico

Fuente: Elaboración propia (2016).

suman al récord de superposiciones. De este modo, el plan parcial reitera las principales transformaciones históricas del sector y amplía su radio de acción de acuerdo con la lectura de las circunstancias actuales.

El plan parcial fue delimitado alrededor de la plaza de mercado, el terminal de transportes, el parque López de Galarza (antes plaza Fallon) y la quebrada El Sillón. Los vértices generados al oriente son producto de la economía de la intervención que aún no ha contado con los recursos necesarios para ser ejecutada (El Nuevo Día, 2015). Por lo tanto, las manzanas 3.3 y 3.6 son excepcionales porque muestran una fuerte relación vial ejercida por la calle 23 entre la plaza de mercado y la carrera quinta y evidencian la ausencia de un análisis morfológico histórico en el plan parcial.

Las consecuencias de esta omisión se manifiestan en los porcentajes históricos de usos del suelo, pues en el plan de 1935 la zona comercial y sus zonas complementarias suman un $24 \%$, el cual se incrementó en la práctica hacia un $62,8 \%$ en 1958 y luego fue reducido a un $13 \%$ con el plan vigente (Figura 18).
El interrogante esencial consiste en el impacto del $49,8 \%$ de comercio que sería desplazado y desintegrado de la plaza de mercado. A ello se suma que el porcentaje de equipamiento entre 1958 y 2011 se conserva y con él la deficiencia identificada en 1958. También se conserva el porcentaje de vivienda, pero se densifica, lo cual se ve representado en un paso del $4 \%$ al $40 \%$ de espacio público. Finalmente, las tendencias porcentuales del plan parcial de 2011 contravienen las vocaciones de los sectores identificadas por el Plan Piloto de 1966 y plantean la supremacía del espacio público de modo similar al de 1935. De ello se deduce la idealidad de la propuesta urbana más reciente por la omisión de las dimensiones de la superposición histórica.

Interpretación de las dimensiones en la superposición

El revelado del ejercicio de superposición de cartografía histórica (Figura 16) conduce a interpretaciones que pueden solucionar algunos problemas de proyectación 


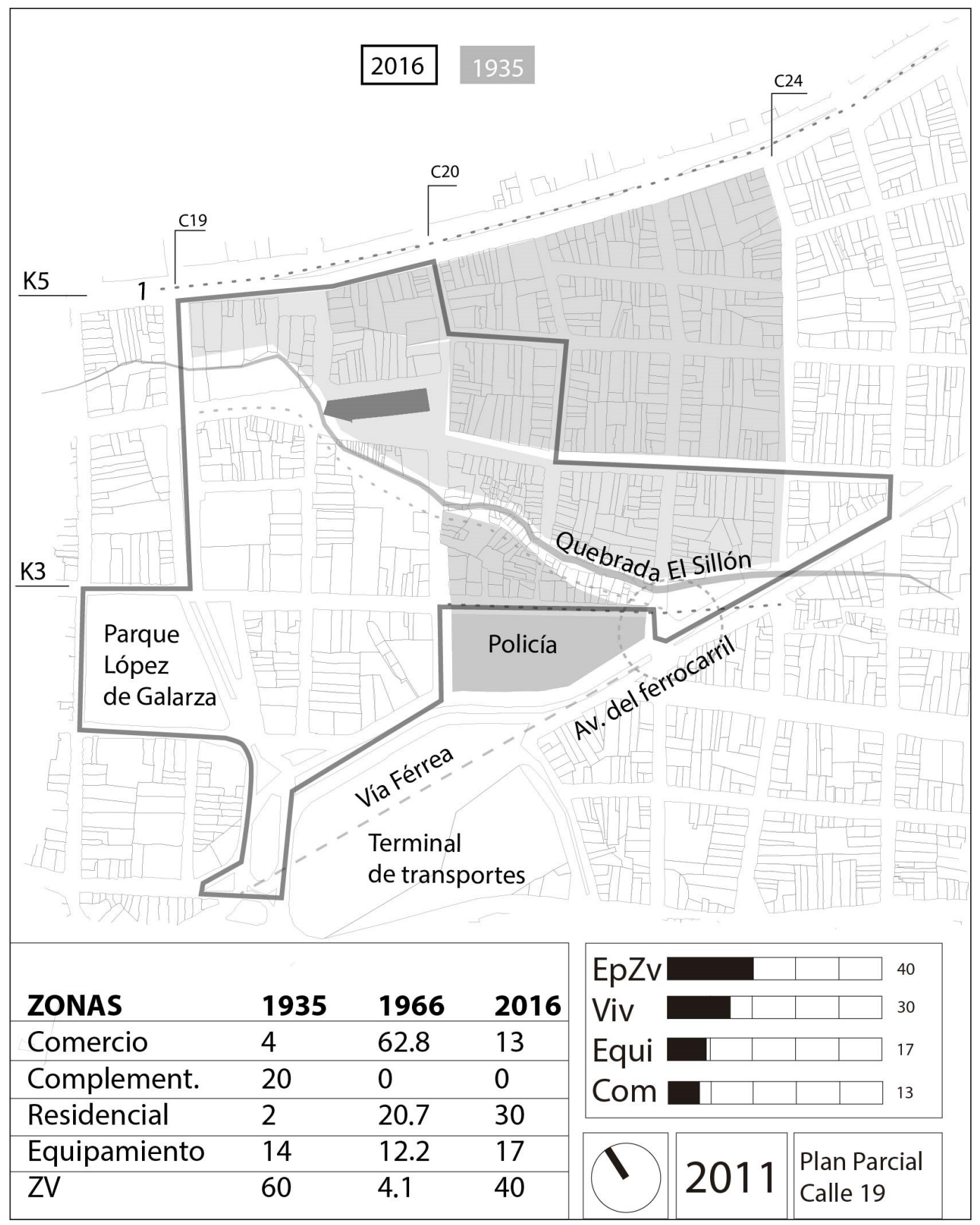

Figura 18 - Comparación de porcentajes entre los planes de 1935, 1958 y 2016

Fuente: Elaboración propia (2016).

en el sector. Se determina que la Calle 19 es una barrera entre el sector del centro y el sector de la calle 21 y no existen elementos ni propuestas de integración. La primera solución es hallar el modelo de integración entre los sectores, el cual se encuentra al observar que la tensión principal es ejercida entre la plaza de mercado y el terminal de transportes a través de la Calle 20, de modo que la conexión se halla en este primer circuito de la Carrera Cuarta, plaza de mercado, Calle 20, terminal de transportes (Figura 19). La vocación de esta línea es el comercio, por lo cual se deben integrar las dinámicas entre el terminal y la plaza, a pesar que los planes vigentes centran la conectividad en la Calle19.

La transformación constante de las manzanas 5.1 y 5.2 se debe a que la morfología urbana no se ha adaptado a la quebrada El Sillón y por lo tanto la ribera, a pesar de estar pavimentada, revela su 


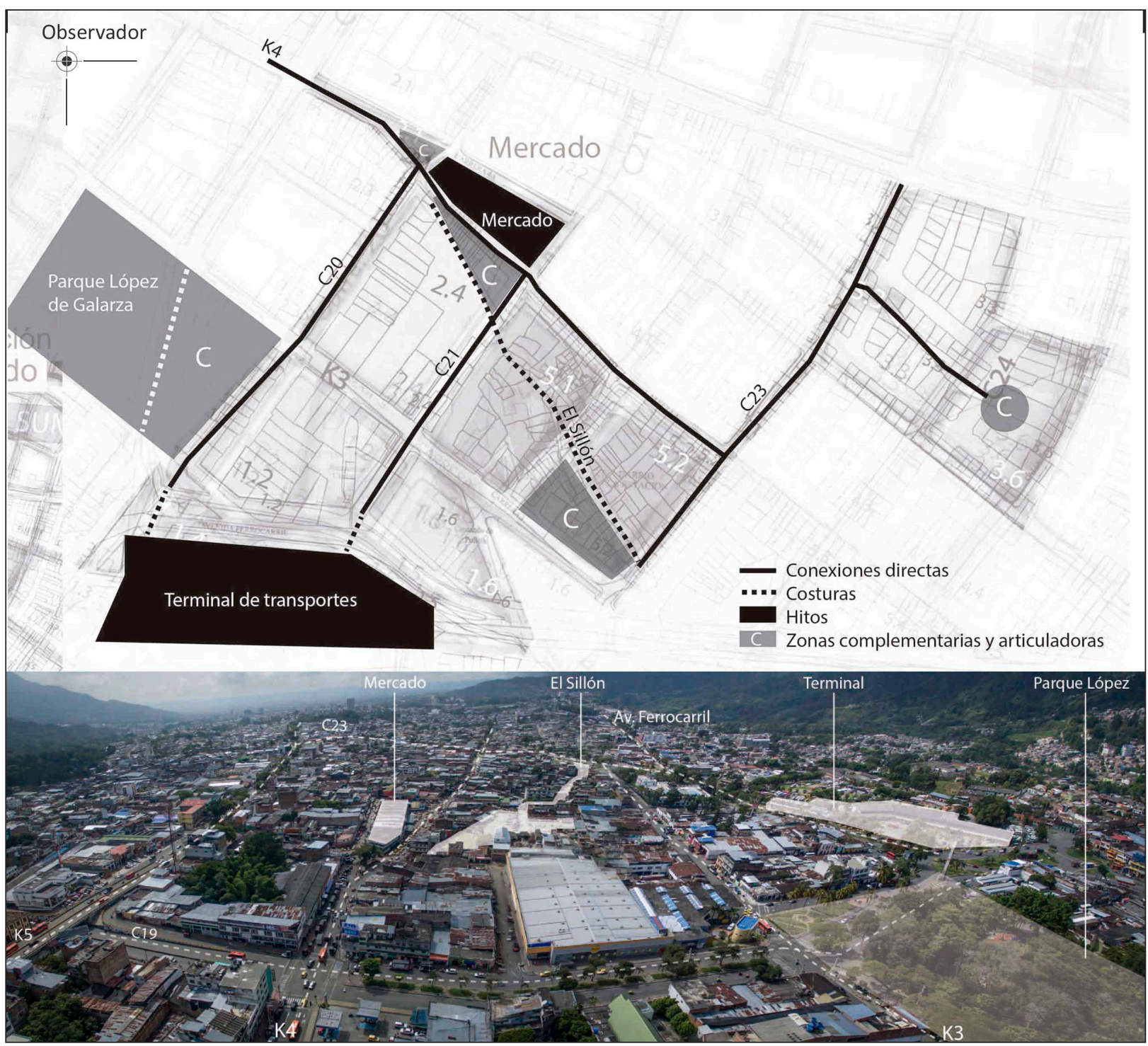

Figura 19 - Gráfica interpretativa de la superposición cartográfica

Fuente: Elaboración propia sobre fotografía de Juan Camilo Aranzález (2016).

huella en los parqueaderos y callejuelas internas de las manzanas (Figura 19). La quebrada conlleva una relación directa entre la plaza de mercado y la Calle 23 como conector comercial entre la plaza de mercado y la Carrera Quinta, ignorado en los planes urbanísticos. La vocación del sector fue interpretada acertadamente desde 1935 como un corredor verde con un concepto paisajístico que permite una vía alterna de integración y transición entre el uso residencial, la plaza de mercado y el comercio complementario de las manzanas 3.3 y 3.6.

Las transformaciones constantes de las manzanas 3.3 y 3.6 corresponden con una escorrentía equivalente al lecho de la quebrada El Sillón, con lo cual se obtienen principios ordenadores para los dos sectores. Finalmente, las zonas complementarias y articuladoras presentan correspondencias entre la morfología y los espacios que a nivel histórico han sido planteados para apoyar los procesos de movilidad y comercio (Figura 19).

\section{Conclusiones}

La técnica de revelado a partir de la superposición de cartografía histórica permite establecer constantes de cambios morfológicos para cuya interpretación se requieren los elementos contextuales presentes en los documentos notariales. Para develar su significado, 
se propone la concepción de un cambio como una operación de inconformismo, una necesidad de ajuste y por lo tanto un acto que devela un paradigma superpuesto de acuerdo con una realidad específica. Esa realidad contiene dos líneas fundamentales. La primera es la idealidad del planeador y la segunda la dinámica económica. La superposición muestra que la dinámica económica prima o se superpone a la idealidad del planeador porque los gobiernos pagan estudios legalmente obligatorios pero no los implementan porque son inconvenientes con las dinámicas inmobiliarias.

Como complemento y explicación, los planeadores no han incorporado la dinámica económica porque sus propuestas han carecido de un estudio de interpretación histórica sobre las transformaciones morfológicas, las cuales son pruebas de una cultura material que guía una sociedad, así que los planeamiento urbanos se observan como negación de esa cultura e imposición de una nueva. Simultáneamente, los conceptos de cicatriz, costura y residuo urbano permiten que el reconocimiento del territorio subyacente ayude a la identificación y validación de las ideas sobre el uso del suelo en cada momento de la historia, de lo cual se pueden generar lineamientos interpretativos, más que impositivos, pues en ello radica el problema analizado.

Finalmente, la desarticulación entre los planes nacionales y los locales evidencia que la idealidad jurídica e infraestructural del desarrollo nacional se impone constantemente a la cultura material local sin que exista claridad sobre el acoplamiento entre las dos instancias, como se constata en el origen de la fragmentación urbana generada por el trazado de la vía del ferrocarril, indiferente al futuro desarrollo de la ciudad. Como resultado, el concepto nacional de progreso violentó el desarrollo urbano, lo cual generó la coexistencia de una economía efervescente y una sociedad con bajos índices de desarrollo humano en medio de un sector fragmentado.

\section{Referencias}

Alba Castro, J. M. (2013). El plano Bogotá Futuro: primer intento de modernización urbana. Anuario Colombiano de Historia Social y de la Cultura, 40(2), 179-208.

Angulo Posse, I. G. (2011). Contribucion de los sistemas de informacion geografica en la formulacion de planes parciales: caso plan parcial calle 19 Ibague (Tesina de maestría en diseño urbano). Universidad Nacional de Colombia, Bogotá.

Arango, S. (1989). Historia de la arquitectura en Colombia. Bogotá: Centro editorial y Facultad de Artes, Universidad Nacional de Colombia.

Arévalo Hernández, D. (1997). Misiones económicas internacionales en Colombia 1930-1960. Revista Historia Crítica, (14), 7-24. Récupéré le 21 julio 2016, de http:// historiacritica.uniandes.edu.co/view.php/462/index. php?id=462

Asociación Colombiana para el Estudio de la Población ACEP. (1975). La población de Colombia. Bogotá: L. Canal y Asociados Ltda. Récupéré le 21 julio 2016, de http:// www.cicred.org/Eng/Publications/pdf/c-c9.pdf

Bogotá. Presidencia de la República. Comité Nacional de Planeación. (1958). Misión de economía y humanismo: estudio sobre las condiciones del desarrollo de Colombia (Vol. I, II). Bogotá: Aedita-Cromos.

Cirer-Costa, J. C. (2011). Geografía fractal de un gran destino turístico. Munich: Munich Personal RePEc Archive. Récupéré le 21 julio 2016, de http://mpra.ub.uni-muenchen. de/33855/

Colombia. Ministerio de Vivienda Ciudad y Territorio. (2012). Planes parciales. República de Colombia. Récupéré le 21 julio 2016, de http://www.minvivienda.gov.co/ POTPresentacionesGuias/Planes\%20parciales.pdf

Delgado, M. (2006). Morfología urbana y conflicto social: las medidas antigueto como políticas de dispersión de pobres. In R. Bergalli, \& I. Rivera Beiras (Eds.), Emergencias urbanas (p. 133-169). Barcelona: Anthropos.

El Nuevo Día. (2015, 15 febrero). Renovación de la 19, un proyecto sin músculo financiero. El Nuevo Día. Récupéré le 21 julio 2016, de http://www.elnuevodia.com.co/ nuevodia/tolima/ibague/247814-renovacion-de-la-19un-proyecto-sin-musculo-financiero

Francel, A. (2013). Cuatro décadas de arquitectura ibaguereña (1904-1940). Ibagué: Universidad de Ibagué.

Francel, A. (2014). Historia de la arquitectura ecléctica en Ibagué, Colombia, 1904-1940 (Tesis doctoral en historia). Universidad Nacional de Tres de Febrero, Buenos Aires.

Francel, A. (2015). Belén: una mujer, un barrio, una historia. Ibagué, 1920-1925. Ibagué: Caza de Libros Editores.

Franco Taipe, A. S. (2014). Costuras urbanas: reconstitución de zonas con usos incompatibles a partir de espacios 
de uso público (Trabajo de titulación, Vol. 1). Pontificia Universidad Católica del Ecuador, Quito. Récupéré le 21 julio 2016, de http://repositorio.puce.edu.ec/bitstream/ handle $/ 22000 / 6656 / 1.2 .000494 . p d f$ ?sequence $=4$

Ibagué. Concejo Municipal. (2000). Acuerdo 116: por medio del cual se adopta el plan de ordenamiento de Ibague y se dictan otras disposiciones. Ibagué.

Ibagué. Concejo Municipal. (2002). Acuerdo 009: por medio del cual se adopta la normativa general de usos, construcciones y urbanizaciones y se dictan otras disposiciones. Ibagué.

Ibagué. Secretaría de Planeación Munical. (2013). Planes Parciales: componente urbano U10: plan de ordenamiento territorial: revisado y ajustado. Ibagué: Alcaldía de Ibagué.

Juaristi Linacero, J. (1984). La teoría de la información en Geografía: aspectos introductorios. Lurralde: Investigación y Espacio, (7), 219-242. Récupéré le 21 julio 2016, de http:// www.ingeba.org/lurralde/lurranet/lur07/07jua/jua07.htm

Leite Trindade, I., \& Dornelas Câmara, A. (2014). El papel del movimiento "economía y humanismo" y de la SAGMACS en Brasil en la construcción de un nuevo método de proyectación urbana. Seminario Internacional de Investigación en Urbanismo, (6), 1-11. Récupéré le 21 julio 2016, de http://upcommons.upc.edu/bitstream/ handle/2099/15965/054_BGT_Leite_Isabella.pdf?sequence=1

Martinez Silva, C. (2016). El plan piloto de Ibagué, 1966. [A. Francel, Entrevistador]. Ibagué.

Moreno Martín, F. J. (2014). Arqueología de la Arquitectura: uan visión conciliadora desde la Historia del Arte. Arqueología de la Arquitectura, (11), 1-19.

Notaría Primera. (1913). Censo poblacional. Caja 403, legajo 4, documento 1, folios 173-280v. Ibagué: Archivo Histórico Municipal.

Notaría Primera. (1918a). Censo poblacional. Caja 441, legajo 2, documento 1, folios 80-201r. Ibagué: Archivo Histórico Municipal.

Notaría Primera. (1918b). Conteo de las cuadras de la ciudad. Caja 408, legajo 18, documento 2, folios 87-190r. Ibagué: Archivo Histórico Municipal.
Notaría Primera. (1918c). Informe de casas de comercio y oficinas particulares. Caja 408, legajo 9, documento 1, folios 530-553r. Ibagué: Archivo Histórico Municipal.

Ramírez Ríos, J. F. (2011). Historia crítica de la planeación urbana en Colombia: una aproximación interpretativa desde los estudios sociales de la ciencia (Tesina de Maestría en Urbanismo). Universidad Nacional de Colombia, Bogotá. Récupéré le 21 julio 2016, de http://www.bdigital.unal. edu.co/5217/1/393266.2011.pdf

Sabaneta. Concejo Municipal. (2009). Acuerdo 22. Por el cual se revisa y ajusta el plan básico de Ordenamiento Territorial para el Municipio de Sabaneta. Sabaneta. Récupéré le 21 julio 2016, de http://cdim.esap.edu.co/BancoMedios/ Documentos\%20PDF/acuerdon\%C2\%BA22de2009.pdf

Sánchez-Mateos García, J. J., Lozano Gallego, A. I., \& Sánchez Quilez, M. P. (2015). Prevención de cicatrices hipertróficas y queloides. Enfermería Ciudad Real. Récupéré le 21 julio 2016, de http://www.enfermeriadeciudadreal.com/ prevencion-de-cicatrices-hipertroficas-y-queloides-462.htm

Sociedad Industrial de Ingeniería y Construcciones SIDEICO. (1935). Informe sobre el plano de Ibagué, levantado en catálogo de arrendatarios de ejidos, áreas de lotes, etc. Ibagué: Honorable Concejo Municipal de Ibagué.

Tarchópulos, D. (2006). Las huellas del plan para bogotá de le corbusier, sert y wiener. Scripta Nova, 10(218). Récupéré le 21 julio 2016, de http://www.ub.edu/geocrit/ sn/sn-218-86.htm

Van der Maas, S. (2011). El diagrama en la arquitectura. DEARQ Revista de Arquitectura de la Universidad de los Andes, (8), 32-43.

Xolalpa, P. M. (2015). Los ferrocarriles mexicanos y sus cicatrices urbanas: transformación, crecimiento y generación urbana a partir del tendido del sistema ferroviario y la permanencia de los trazos. Estudios sobre Conservación, Restauración y Museología, 1, 27-40. Récupéré le 21 julio 2016, de https://www.revistas.inah.gob.mx/index.php/ estudiosconservacion/article/view/5443/6021

Recibido: Mayo 19, 2016

Aprobado: Jul. 11, 2016 